\title{
Metodologia di rilievo della rugosità superficiale del suolo con Pole Aerial Photography (PAP)
}

\author{
Paolo Bazzoffi, ${ }^{1}$ Roberta Gentile, ${ }^{2}$ Andrea Rocchini ${ }^{1}$ \\ ${ }^{1}$ CREA-ABP, Centro di Ricerca per l'Agrobiologia e la Pedologia, Consiglio per la ricerca in agricoltura e l'analisi dell'economia \\ agraria, Firenze \\ 2Università degli Studi di Siena, tirocinante del Master Universitario di II Livello in Sistemi Informativi Territoriali e \\ Telerilevamento, Siena, Italia
}

Autore corrispondente: Paolo Bazzoffi

E-mail: paolo.bazzoffi@@crea.gov.it

Parole chiave: Metodologia; condizionalità; rugosità superficiale; PAP.

Lavoro svolto nell'ambito del Progetto MO.NA.CO. (Rete di monitoraggio nazionale dell'efficacia ambientale della condizionalità e del differenziale di competitività da essa indotto a carico delle imprese agricole) finanziato dal Ministero delle Politiche Agricole, Alimentari e Forestali (MiPAAF) nell'ambito del Programma Rete Rurale Nazionale nel contesto dell'Azione 1.2.2 "Laboratori interregionali per lo sviluppo" del Programma Operativo denominato "Rete Rurale Nazionale 2007 - 2013.

Redazione a cura di Paolo Bazzoffi.

Ringraziamenti: Gli Autori ringraziano l'operatore tecnico del CREA-ABP Giorgio Moretti per l'assistenza fornita nei rilievi PAP in campagna.
(C) Copyright P. Bazzoffi, 2015

Licenziatario PAGEPress, Italy

Italian Journal of Agronomy 2015; 10(s1):722

doi:10.4081/ija.2015.722

Questo articolo è distribuito secondo i termini della licenza Noncommercial Creative Commons Attribution (by-nc 3.0) che permette qualsiasi uso non commerciale, la distribuzione e la riproduzione con qualsiasi mezzo, a condizione che l'autore (autori) originale(i) e la fonte siano accreditati.

\section{Riassunto}

La valutazione della rugosità superficiale del suolo è importante in agricoltura sotto molteplici aspetti: nello studio delle relazioni tra le rugosità della superficie e volume massimo di stoccaggio di acqua nelle depressioni della superficie per lo sviluppo di modelli idrologici da utilizzare nelle strategie di conservazione del suolo e dell'acqua; ogni qualvolta occorra definire lo stato di amminutamento delle zolle determinato dagli strumenti agricoli di lavorazione del suolo in relazione all'umidità e alle caratteristiche fisiche del suolo.

Lo scopo di questo lavoro è stato quello di calibrare una nuova metodologia di misura della Rugosità Superficiale del Suolo (Random Roughness), basata sull'analisi di immagini stereoscopiche ricavate con Metodologia PAP (Pole Aerial Photography). La nuova metodologia si pone due obiettivi innovativi fondamentali: misurare la Rugosità superficiale su ampie aree campionarie, al fine di una migliore rappresentazione delle superfici; ricavare i valori di rugosità con una tecnologia speditiva a basso costo.

\section{Introduzione}

La valutazione della rugosità superficiale del suolo a fini modellistici dell'erosione può essere acquisita attraverso diversi strumenti. Generalmente vengono usati profilimetri manuali ad aghi o elettronici a laser, ma il loro impiego è molto laborioso e lento, inoltre fornisce risultati su piccole superfici e quindi poco rappresentative delle aree per le quali si applicano i modelli. Anche se la stereo-fotogrammetria non è usata abitualmente per i rilievi topografici di alta precisione, negli ultimi tempi viene utilizzata sempre più spesso. Ciò è dovuto ai progressi delle prestazioni dei sensori CCD (Charge-Coupled Device) delle macchine fotografiche digitali e allo sviluppo di programmi che consentono un approccio più user friendly grazie a metodi automatici di stereocorrelazione. La fotogrammetria da aeromobili a bassa quota fornisce DEM (Digital Elevation Model) in griglie millimetriche, con una precisione sulla posizione sub-millimetrica quindi comparabile al laserscan ma l'accuratezza sulle quote non raggiunge gli stessi risultati (Taconet e Ciarletti, 2007). Nella modellistica dell'erosione del suolo occorre una valutazione quanto più possibile precisa della rugosità superficiale, unitamente alla velocità di acquisizione e a costi accettabili. Con questo studio viene presentata una metodologia per la valutazione della rugosità superficiale per mezzo di stereocoppie fotografiche zenitali acquisite tramite macchina fotografica sospesa a un palo e comandata da terra. Si è quindi valutata l'accuratezza delle quote utilizzando questo metodo speditivo ed economico. 


\section{La Random Roughness nella tecnologia USLE- RUSLE di previsione dell'Erosione Idrometeorica}

Il modello predittivo USLE di Wischmeier e Smith (1978) permette la stima delle perdite di suolo da erosione laminare e incanalata (rigagnoli). Renard et al. (1997) hanno successivamente aggiornato il modello in RUSLE (Revised Universal Soil Loss Equation) per un miglior adattamento di questo strumento predittivo anche su terreni a morfologia complessa.

La tecnologia USLE/RUSLE consiste in un set di equazioni che stimano la perdita di suolo e la produzione di sedimento derivanti dai processi di erosione per rigagnoli e laminare (rill-interril erosion). Essa deriva dallo studio dei processi erosivi su un numero elevato di anni di osservazioni parcellari (più di 10000) sotto l'influenza di pioggia naturale 0 simulata ed è stata molto ben validata e documentata da numerosi studiosi.

I modelli USLE/RUSLE oltre ad essere ancor oggi le più usate a fini applicativi pratici e per studi territoriali, ben rappresentano i fattori principali che determinano l'erosione del suolo.

Il modello RUSLE mantiene la struttura del modello USLE, come segue:

$$
\mathrm{E}=\mathrm{R} \times \mathrm{K} \times \mathrm{LS} \times \mathrm{C} \times \mathrm{P}
$$

L'erosione laminare e per rigagnoli $\mathrm{E}\left(\mathrm{t} \mathrm{ha}^{-1} \mathrm{anno}^{-1}\right)$ è proporzionale ad un parametro, detto erosività della pioggia e del ruscellamento $\mathrm{R}(\mathrm{MJ} \mathrm{mm}$ $\left.\mathrm{h}^{-1} \mathrm{ha}^{-1} \mathrm{y}^{-1}\right)$. Il fattore erodibilità del suolo $\mathrm{K}\left(\mathrm{t}\right.$ ha h ha $\left.\mathrm{h}^{-1} \mathrm{MJ}^{-1} \mathrm{~mm}^{-1}\right)$ rappresenta la perdita di suolo per unità di $\mathrm{R}$, misurata su parcella standard lunga 22,13 m, con il 9\% di pendenza, lavorate nel senso della pendenza e mantenute a maggese nudo. Il fattore LS (fattore topografico) accorpa il fattore lunghezza e pendenza. Il fattore L (adimensionale) tiene conto dell'effetto della lunghezza della pendice sull'erosione e rappresenta il rapporto fra le perdite di suolo relative ad una data lunghezza dell'appezzamento rispetto a quello della parcella standard, avente lunghezza $22,13 \mathrm{~m}$. Il fattore pendenza $S$ (adimensionale) è il rapporto tra le perdite di suolo della parcella in esame e quella della parcella standard avente pendenza del $9 \%$. C (adimensionale) è il fattore relativo alla coltura e alle tecniche colturali, esso varia fra 0 e 1 ed esprime il rapporto fra l'erosione di una data coltura e tecnica colturale con quello del maggese nudo. $\mathrm{P}$ (adimensionale) rappresenta il fattore relativo alle pratiche per conservare il suolo dall'erosione (esso varia fra 0 e 1), come rapporto fra erosione derivante da una data pratica conservativa e quello ottenibile dal terreno nudo lavorato a rittochino.

Poiché il modello è una moltiplicazione di fattori, è sufficiente che uno dei valori inseriti sia prossimo a zero affinché anche il risultato finale diminuisca drasticamente. Tralasciando la descrizione dei parametri R e K, che esula dalla finalità del presente lavoro, si focalizza l'attenzione sul fattore C "Copertura del suolo", che fra le sue componenti comprende il fattore "Rugosità del suolo".

\section{II multifattore C del modello RUSLE}

Nel modello RUSLE il fattore copertura e gestione "C" rappresenta l'effetto sulla perdita di suolo della vegetazione, della copertura del suolo, della biomassa radicale, dei residui incorporati, della rugosità di superficie, dell'umidità del terreno, della gestione del suolo e delle pratiche gestionali annuali di lotta all'erosione. Come detto, il fattore $\mathrm{C}$ è il rapporto fra la perdita di suolo in presenza di una determinata coltura/gestione del suolo e l'erosione che si otterrebbe dalla parcella stan- dard in condizioni di suolo preparato per la semina e privo di vegetazione. È facilmente intuibile che il fattore $\mathrm{C}$ è di notevolissima importanza nella valutazione dell'impatto dell'agricoltura sul suolo, essendo relativo al tipo di coltivazione e di gestione.

Poiché l'erosione varia con il variare dell'erosività della pioggia e della morfologia della parte aerea delle piante, nel valutare il valore annuale di C dovranno essere considerati i cambiamenti nella morfologia della pianta durante l'anno e gli effetti della successione colturale, del livello di rendimento della coltura (da cui dipende anche la quantità di residui lasciati sul suolo dalla coltura), della lunghezza del periodo di crescita, delle pratiche agricole, della gestione dei residui e della possibile distribuzione delle piogge per una data stazione (Schwab et al., 1981). L'azione mitigatrice della copertura vegetale riguardo alla pioggia varia quindi in funzione del complesso di pratiche e condizioni che determinano il sistema agricolo. Nella USLE (Wischmeier (1975) e Mutchler et al. (1982) hanno indicato che l'impatto della coltura e della gestione agricola sull'erosione può essere diviso in sotto-fattori per calcolare i rapporti di perdita di suolo per ogni periodo del ciclo vegetativo-colturale e l'erosione attesa dalla parcella standard.

Nella RUSLE i fattori per il calcolo del rapporto di perdita di suolo sono stati modificati da Laflen et al. (1985) e da Weltz et al. (1987) e si riferiscono: i) all'uso del suolo precedente alla coltura (PLU); ii) alla copertura del suolo determinata dalla parte aerea delle piante (CC); iii) alla copertura a contatto del suolo (SC); iv) alla rugosità (SR) e E) all'umidità del suolo (SM).

Basandosi sulle nuove descrizioni della coltura e dell'influenza delle pratiche agricole sulle perdite di suolo, Laflen et al. (1985) hanno proposto il calcolo dei rapporti di perdita di suolo nel seguente modo:

$$
\mathrm{SLR}=\mathrm{PLU}-\mathrm{CC} * \mathrm{SC} * \mathrm{SR}-\mathrm{SM}
$$

dove SLR è il rapporto di perdita di suolo (soil-loss ratio) per una data condizione. Tralasciando la trattazione dei fattori PLU, SC e SM, si descrive in dettaglio il sotto-fattore SR, che è oggetto del presente studio.

\section{Sottofattore SR - Rugosità (scabrezza) superficiale del suolo}

La scabrezza della superficie del suolo influenza l'erosione sia direttamente (Cogo et al. 1984) diminuendo la velocità del deflusso e riducendo la sua capacità di trasporto, sia indirettamente agendo sull'efficacia dei residui colturali nel proteggere il suolo. Essa viene considerata nel fattore $\mathrm{C}$ attraverso il sottofattore SR (Surface Roughness) ed è definita come la deviazione standard delle quote della superficie del suolo depurate dai cambiamenti della superficie dovuti alla pendenza o le depressioni non casuali (tracce lasciate dal trattore, solchi effettuati dall'uomo, ecc.).

Un'alta rugosità della superficie incrementa l'infiltrazione e diminuisce il grado di sigillamento del suolo dovuto all'impatto delle gocce (Sumner e Stewart, 1992). Al contrario un suolo finemente amminutato, 0 addirittura polverizzato da eccessive lavorazioni, sarà soggetto a sigillare velocemente e conseguentemente ad avere bassi tassi di infiltrazione, generando deflusso superficiale. La scabrezza dipende principalmente dall'attrezzo agricolo utilizzato per la lavorazione, ma anche dalla velocità del trattore e dalle condizioni del suolo prima della lavorazione (tipo di lavorazione effettuata in precedenza, umidità, consolidamento, sostanza organica, uso del suolo precedente, ecc.).

La condizione base, cui corrisponde un valore $S R=1$, si riferisce a un suolo sul quale sia presente una coltivazione in fase di crescita nel quale la zollosità del letto di semina è stata stemperata da piogge di intensità moderata.

Alla fine del ciclo colturale si assume che il valore SR sia diminuito fino al valore 0,24 a causa della normale gestione agricola e dell'azione della pioggia. Il sottofattore SR viene calcolato secondo la seguente 
espressione:

$$
\mathrm{SR}=e^{\left[-0,026\left(R_{\mathrm{u}}-6,1\right)\right]}
$$

dove $R_{u}(0$ RR) è la rugosità superficiale prima dell'operazione agricola considerata. L'indice di rugosità più noto e utilizzato è sicuramente la random roughness (RR) che nella formulazione definitiva di Currence e Lovely (1970) è espresso come la deviazione standard delle altezze relative del profilo o della superficie campionate secondo un passo o maglia regolare:

$$
\mathrm{RR}=\sqrt{\sum_{t=1}^{n}\left(Y_{i}-\mu_{y}\right) / n}
$$

dove: $\boldsymbol{n}$ è il numero delle altezze relative campionate; $\mu_{\mathrm{y}}$, la media aritmetica delle altezze campionate; $\boldsymbol{Y}_{\boldsymbol{i}}$ il valore di ogni singola altezza relativa nel profilo (Bazzoffi, 2006).

\section{La Low-Altitude Aerial Photography}

La Pole Aerial Photography (PAP), o fotografia da palo, fa parte di una serie di metodologie, utilizzate sin dai primi del novecento, in particolare in archeologia, chiamate Low-Altitude Aerial Photography (LAAP). Le LAAP (chiamate anche low-level, large-scale o close-range aerial photography) permettono di ottenere foto aeree a bassissima quota in grado di fornire una ricchezza di particolari difficilmente ottenibile con le foto aeree tradizionali da aeroplano.

Le tecniche fotogrammetriche che utilizzano un palo come sostegno si avvalgono di macchine fotografiche, spesso non metriche, applicate ad un'ampia gamma di mezzi, che possono essere dei semplici sostegni con basi poggiate a terra come pali, gru o scale che consentono di arrivare mediamente ad un'altezza massima di $10 \mathrm{~m}$. I costi di realizzazione delle fotografie da palo sono molto più bassi di quelli determinati dall'impiego sia di aeroplani sia dall'impiego di droni. La metodologia PAP è stata impiegata sin dall'inizio del XX secolo per documentare scavi archeologici e generare planimetrie a grande scala (Verhoeven, 2009), mentre non risulta essere stata precedentemente applicata all'analisi della stima rugosità del suolo negli stessi termini delle presente indagine. Nel nostro studio la strumentazione PAP è stata realizzata attraverso un semplice palo telescopico il quale, data la necessità di ricorrere ad un metodo speditivo, volutamente non presentava al momento del rilievo particolari accorgimenti di stabilizzazione. Le indagini effettuate si sono sviluppate in due campagne consecutive di rilievo in campo, nelle quali sono stati acquisiti tutti gli elementi necessari alla successiva elaborazione fotogrammetrica (foto, punti GPS, ecc.). Nella prima campagna si è valutata l'affidabilità del metodo prendendo in esame le quote GPS di alcuni punti noti e confrontandole con quelle degli stessi punti restituiti a video tramite stereo-coppie di foto PAP. Nella seconda campagna si sono messi a confronto i profili ottenuti con profilimetro ad aghi e i profili ottenuti in ambiente GIS dal DEM estratto con la stereoscopia. Da entrambe le tipologie di profilo sono state poi calcolati e comparata i valori di rugosità (RR).

\section{Materiali e metodi}

La strumentazione di sostegno della fotocapera è stata appositamente realizzata da un'officina di meccanica di precisione di Firenze e consiste in un palo telescopico da telecomunicazioni (prodotto dalla Ditta Arno, Pontedera, PI) in fibra di vetro e poliestere, costituito da 8 ele- menti lunghi ciascuno m 1,40. Il diametro della sezione che appoggia a terra è $58 \mathrm{~mm}$, mentre la sezione più piccola (in alto, alla quale è applicata la macchina fotografica) ha un diametro di $18 \mathrm{~mm}$ (Figura 1). All'estremità del palo è presente un supporto in alluminio (Figura 2a) dove poter fissare la macchina fotografica la quale grazie a degli snodi può assumere una posizione nadirale rispetto al geoide. Per le riprese fotografiche è stata utilizzata una Canon PowerShot G7 (Figura 2b), le cui caratteristiche principali sono riportate in Tabella 1. Si tratta di una macchina digitale commerciale non metrica, della quale non si possie-

Tabella 1. Estratto delle specifiche tecniche della macchina

\begin{tabular}{|c|c|}
\hline \multicolumn{2}{|l|}{ Sensore immagine } \\
\hline & 1/1,8" CCD \\
\hline & Pixel effettivi $\quad$ Ca. $10.0 \mathrm{M}$ \\
\hline & Tipo filtro colore $\quad$ Colori primari \\
\hline \multicolumn{2}{|l|}{ Obiettivo } \\
\hline Lunghezza focale & $\begin{array}{l}7,4-44,4 \mathrm{~mm} \\
\text { (equivalente a } 35-210 \mathrm{~mm} \text { in formato } 35 \mathrm{~mm} \text { ) }\end{array}$ \\
\hline Stabilizzatore ottico & Sì (tipo shift) \\
\hline \multicolumn{2}{|c|}{ Pixel registrati / compressione } \\
\hline Dimensione immagine & $\begin{array}{l}\text { (L) } 3648 \text { x 2736, (M1) } 2816 \text { x 2112, (M2) } 2272 \\
\times 1704,(\mathrm{M} 3) 1600 \times 1200, \text { (S) } 640 \times 480 \\
\text { (W) } 3648 \times 2048\end{array}$ \\
\hline Compressione & Superfine, Fine, Normal \\
\hline
\end{tabular}
fotografica digitale non metrica Canon PowerShot G7.

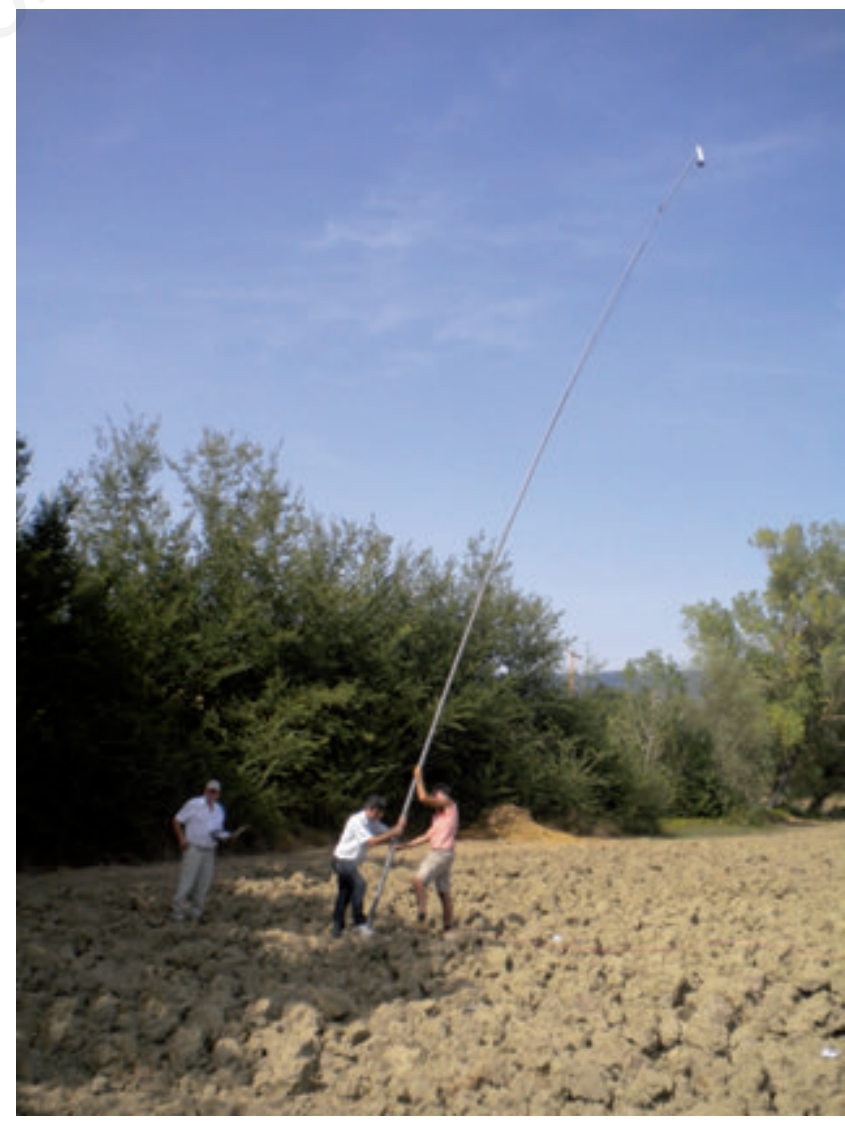

Figura 1. Pole Aerial Photography, Fagna (FI). 
de il certificato di calibrazione. La scelta della macchina fotografica è ricaduta su un modello che consentiva di salvare il file in formato ROW e che permetteva la gestione in remoto dello scatto. Altra caratteristica importante che ha guidato alla scelta di questa fotocamera è stata la presenza dello stabilizzatore ottico d'immagine, capace di contrastare le vibrazioni involontarie della fotocamera, permettendo una maggiore nitidezza nei dettagli.

La camera è stata collegata ad un notebook tramite un cavo USB ed un fermo impediva al cavo stesso di scivolare davanti all'obiettivo. Per il rilievo GPS dei punti è stato utilizzato un Leika 1200 GPS+GNNS (Figura 3), un ricevitore GPS geodetico a doppia frequenza L1 ed L2 in configurazione tipo con antenna esterna. La stazione master in comunicazione radio con la stazione rover, era applicata ad un treppiedi telescopico in alluminio dotato di tricuspide con piombino ottico alla base dell'antenna. La stazione rover era collegata tramite cavo all'antenna sistemata su una palina di alluminio con punta di acciaio e bolla. I punti di appoggio sono consistiti in picchetti da campeggio (Figura 4) ai quali sono state applicate delle piastrine in plastica bianca recanti i
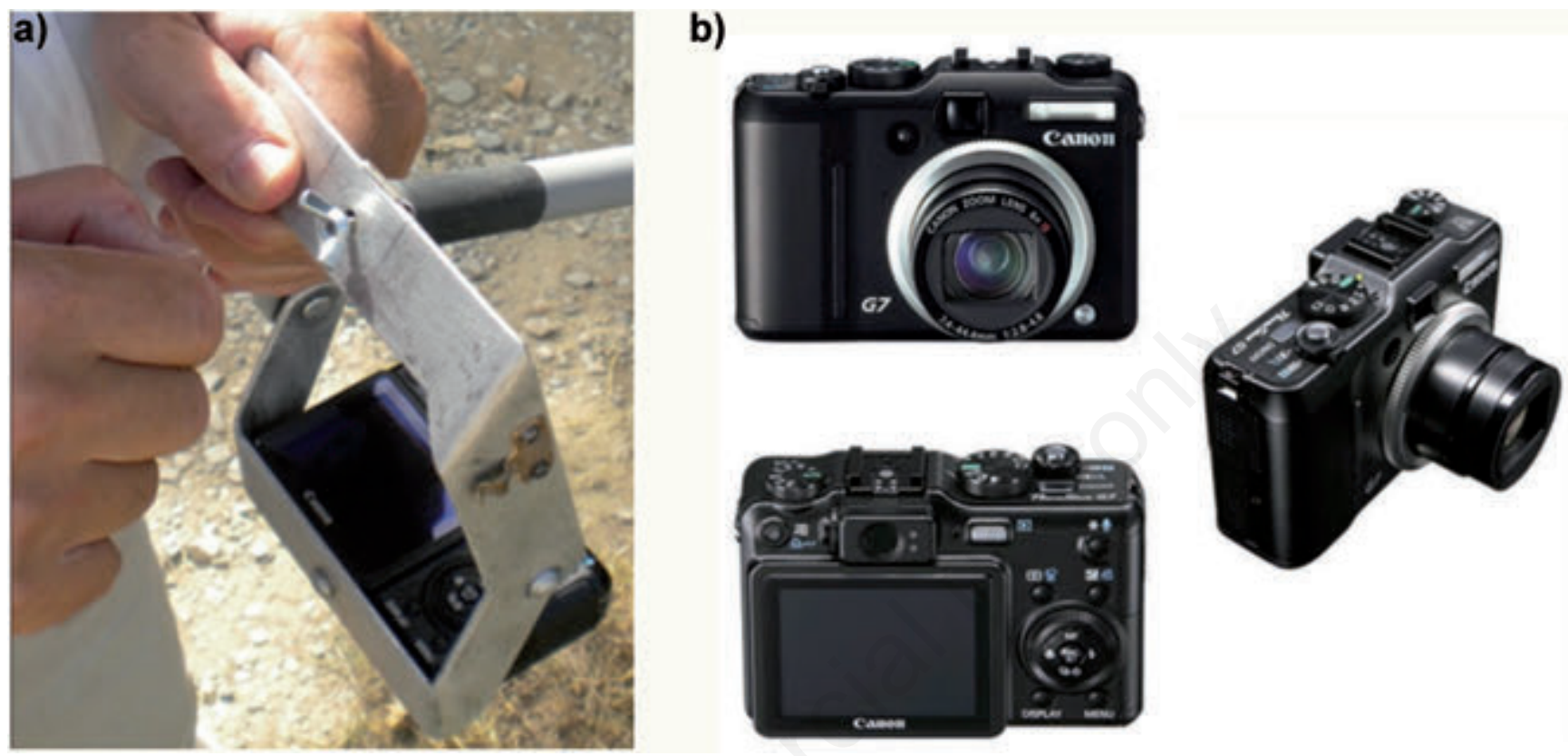

Figura 2. a) Supporto in alluminio presente all'estremità del palo. b) Fotocamera digitale Canon PowerShotG.

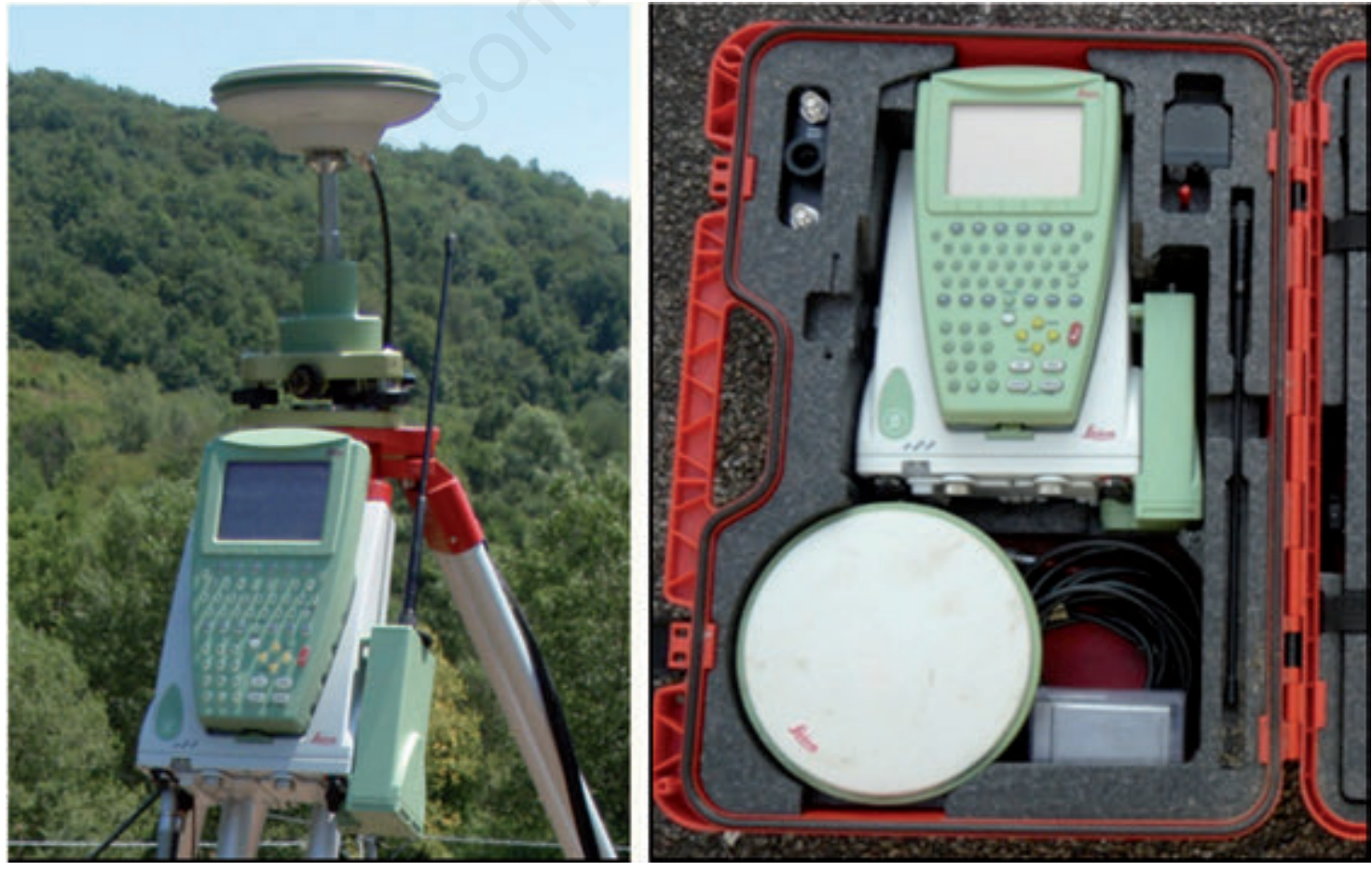

Figura 3. GPS Leika 1200 +GNNS montato su treppiedi in alluminio e a destra nella valigetta contenitore. 
numeri da uno a venti, stampati in dimensioni tali da poter essere ben distinguibili dall'alto. I punti di controllo erano formati da puntine da disegno colorate. Una cornice quadrata di profilato metallico, che definisce al suo interno un'area di un metro quadrato, ha costituito l'area bersaglio, su cui focalizzare le verifiche. Il rilievo dei profili in campo è stato effettuato con un profilimetro ad aghi (Figure 5 e 6) costituito da una cornice metallica che sostiene un pannello di policarbonato alveolare trasparente. All'interno degli alveoli scorrono aghi di alluminio di $8 \mathrm{~mm}$ di diametro distanziati tra loro di $13 \mathrm{~mm}$. Un fermo posizionato sul bordo inferiore del pannello evita agli aghi di fuoriuscire dalla cornice nel momento del posizionamento verticale sulla sezione di terreno da rilevare.

\section{Procedure fotogrammetriche}

Il processo fotogrammetrico consiste in un insieme di operazioni che consentono di giungere alla definizione delle coordinate tridimensionali di un punto (Salvini, 2011) attraverso la ricostruzione rigorosa della corrispondenza geometrica tra l'immagine e l'oggetto che lo ha generato (Gomarasca, 2004).

Nella nostra indagine, poiché sono stati presi in considerazione processi interamente digitali sia di presa dei fotogrammi che di analisi, si tratta più precisamente di fotogrammetria digitale. Il processo fotogrammetrico può essere suddiviso nelle seguenti fasi:

- La Presa dei Fotogrammi

- Appoggio: determinazione di una serie di punti per le successive
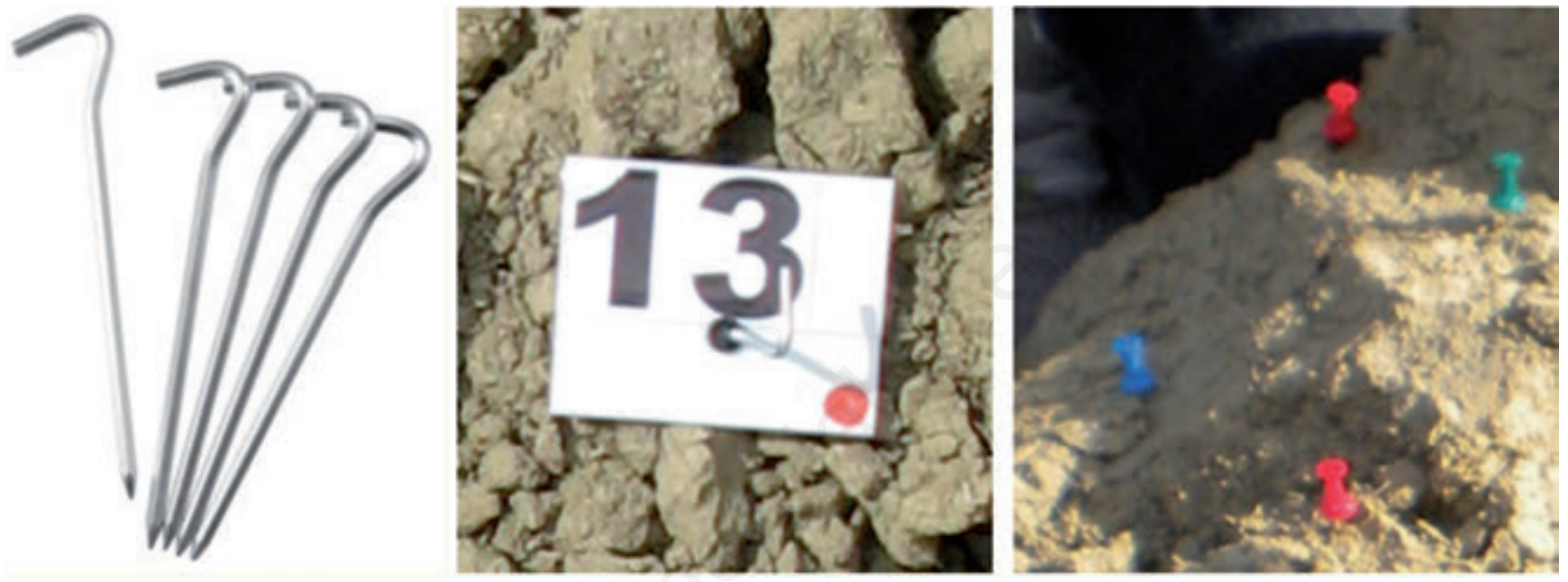

Figura 4. Picchetti da campeggio, piastrine di plastica, puntine da disegno.

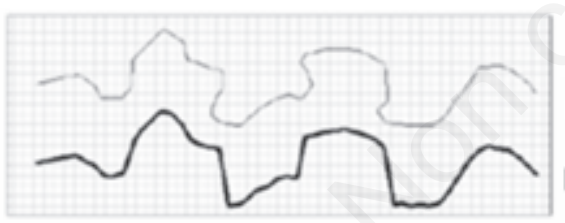

profilo reale profilo riprodotto

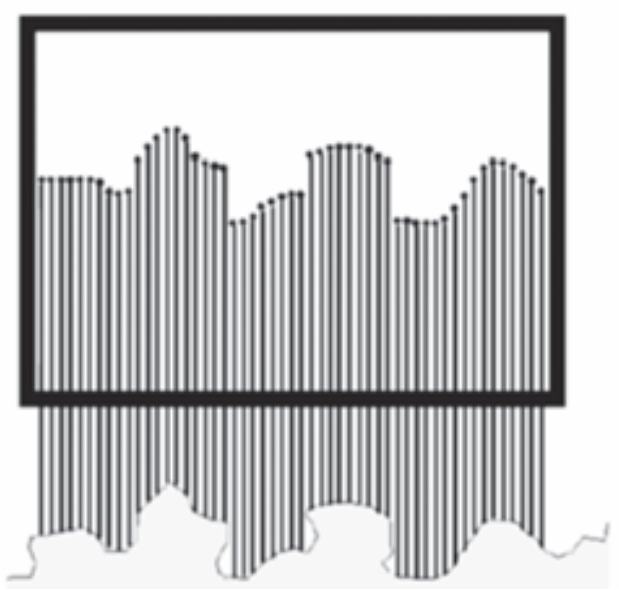

Figura 5. Schema del profilimetro ad aghi.

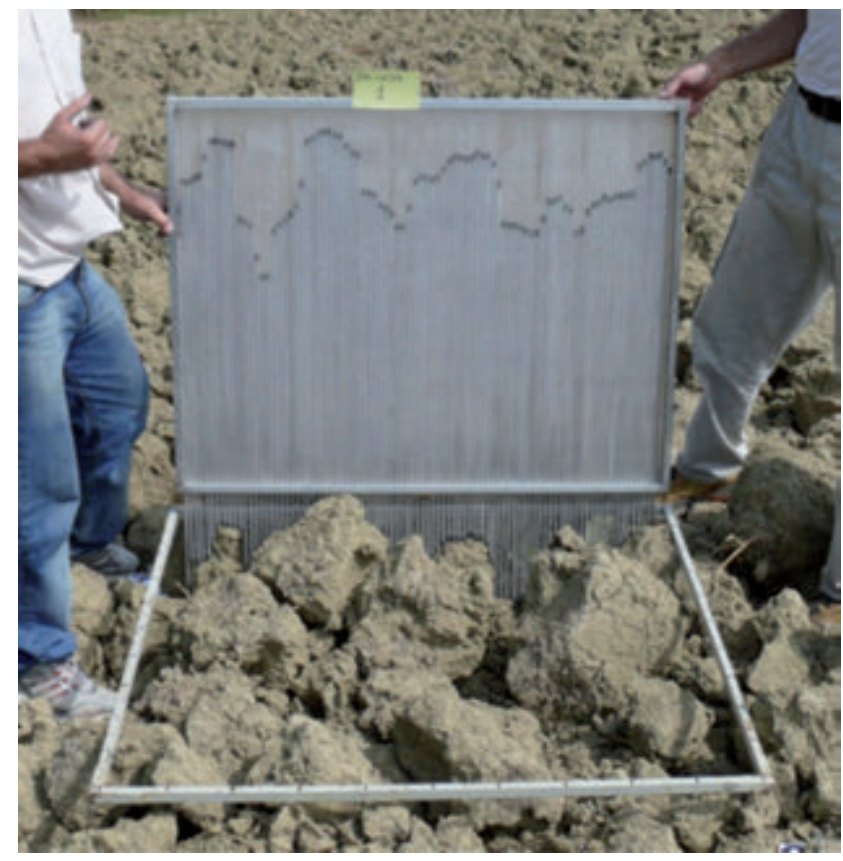

Figura 6. Rilievo dei profili superficiali con profilimetro ad aghi. 
operazioni di orientamento

- Orientamento: ricostruzione indiretta dell'assetto spaziale dei fotogrammi

- Restituzione: determinazione delle coordinate tridimensionali dei punti oggetto del rilievo

\section{Presa dei Fotogrammi e Appoggio}

\section{Prima sessione}

La presa dei fotogrammi ha previsto prima di tutto la scelta del luogo più adatto all'esperimento. Tale scelta è ricaduta su un terreno di un'azienda del CRA-ABP situato nei pressi si Scarperia in località Fagna (FI) (Figura 7). Tale appezzamento era stato arato in Agosto e presentava zollosità elevata.

Il rilievo è iniziato con il posizionamento della stazione master su un punto georeferenziato all'interno dell'azienda sperimentale (inquadramento GPS del caposaldo denominato "stalla", agganciandosi al vertice IGM95:106621 denominato Le Maschere). Arrivati nella zona prescelta il primo passo è stato quello di sistemare a terra un cordino rosso mantenuto in tensione tra due picchetti. Questo cordino è stato utile per mantenere il palo il più possibile allineato con la linea di volo e per la sistemazione dei picchetti dei punti di appoggio. La costanza della linea di volo permetteva di limitare eventuali difetti di deriva 0 sbandamento del punto di presa dovuto ad un non corretto posizionamento del palo. Conoscendo invece l'abbracciamento della foto è stato possibile sistemare i picchietti ai lati del cordino in maniera tale da che non ricadessero fuori dell'area di presa e che ogni foto comprendesse più di tre GCP non allineati.

Successivamente è stato posizionato al centro della linea di volo il frame metallico dentro il quale sono state disposte in maniera uniforme le 100 puntine da disegno.

\section{Rilievo GPS dei punti di appoggio}

Il rilievo GPS è stato effettuato in modalità RTK sostando cinque epoche per ogni GCP. Un operatore posizionava la palina sul punto e, una volta in bolla, dava il segnale ad un altro operatore che acquisiva la registrazione delle coordinate (Figura 8). Lo stesso tipo di rilievo è stato effettuato per le puntine da disegno, in questo caso, data la delicatezza dell'operazione, gli operatori erano tre, uno reggeva la punta della palina sul punto, uno metteva in bolla e uno acquisiva i dati.

Terminato il rilievo GPS sono state acquisite le foto. Sistemata la Canon sull'apposito supporto il palo è stato portato alla sua massima estensione (Figura 9); quindi è stato issato in posizione verticale e posizionato sul primo step della linea di volo. Il palo veniva mantenuto nella posizione corretta grazie ad una bolla applicata all'elemento basale del palo stesso (Figura 10).

Per ogni stazione di presa sono state scattate più foto in maniera da poter scegliere le migliori in termini di errori di beccheggio e rollio anche se prima di scattare le foto si prestava attenzione a che la macchina oscillasse il meno possibile (Figura 11). La base aerea, definita come la distanza fra un punto di presa e il successivo, era di circa un metro e questo ha consentito di avere un overlap fra i fotogrammi di circa l' $80 \%$.

\section{Seconda sessione di rilievo}

La seconda sessione di rilievo si è svolta nelle medesime condizioni e con la medesima strumentazione della prima per ciò che riguarda il rilievo fotografico e dei punti GPS.

L'area oggetto di studio era rappresentata dal medesimo frame metallico, ma questa volta l'obbiettivo non era misurare le quote di punti prestabiliti, ma valutare la corrispondenza tra profili di suolo rilevati con metodi tradizionali e quelli estratti da metodi digitali. Il rilievo dei profili è iniziato definendo la griglia di rilevo. Sulla cornice del frame sono stati posizionati dei marker alla distanza di $10 \mathrm{~cm}$ l'uno dall'altro, permettendo di ottenere 18 tracce come mostrato in Figura 12.

In campo, il profilimetro è stato poggiato sulle sezioni prestabilite e sbloccando l'apertura dalla quale fuoriuscivano, gli aghi scorrevano verso il basso arrestandosi quando l'estremità libera giungeva a toccare le zolle. La parte superiore degli aghi colorata di nero tracciava il profilo del suolo (Figura 6). Un operatore scattava poi una foto al pannello cercando di restare nei suoi confronti il più possibile perpendicolare $\mathrm{e}$ centrale. Infine, per cercare di rendere agevole il riconoscimento del profilo in ambito digitale, sono state rilevate le posizioni GPS degli gli spigoli del frame.

\section{Post-processing}

Il post-processing dei dati GPS è stato eseguito con il software Leika Geo Office. L'accuratezza globale delle coordinate è risultata mediamente pari a $0,017 \mathrm{~m}$.

\section{Orientamento dei fotogrammi}

Le immagini prodotte da una macchina fotografica, metrica o non metrica, hanno la caratteristica di essere state generate attraverso uno

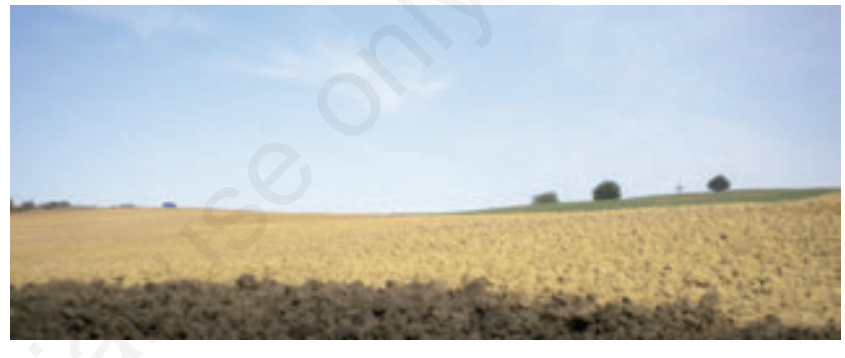

Figura 7. Appezzamento scelto come area studio, situato a Scarperia località Fagna (FI).

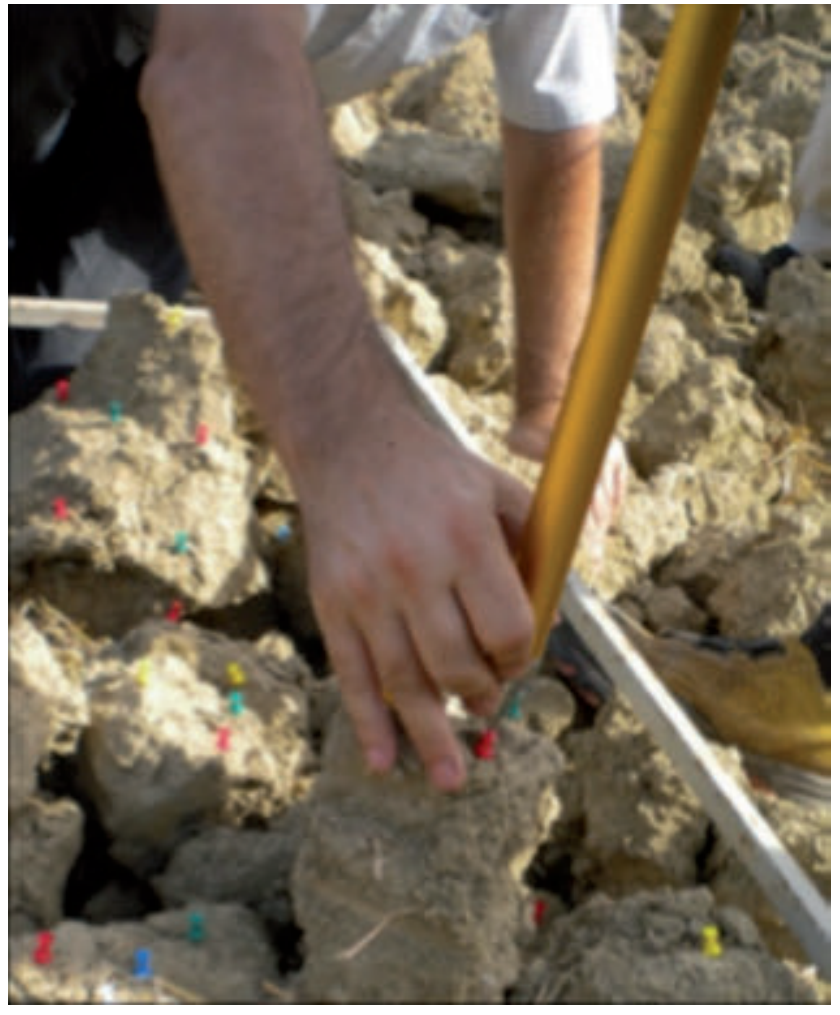

Figura 8. Rilievo dei punti di controllo. 
schema geometrico unico per l'intero spazio oggetto: ogni punto oggetto $P$ ha un suo corrispettivo Punto Immagine univocamente determinato attraverso leggi di trasformazione i cui parametri sono identici per ogni punto.

L'identificazione di tali parametri costituisce l'orientamento dei fotogrammi. Ed in particolare:

- Tre parametri per l'orientamento interno: le coordinate $\xi_{0}$ e $\eta$ del punto principale e la distanza focale $\mathrm{c}$ individuano la posizione del centro di proiezione sul piano dell'immagine e definiscono l'orientamento interno del fotogramma (a cui bisogna associare anche la distorsione radiale).

- Sei parametri di orientamento esterno: le coordinate Xo, Yo e Zo del centro di presa rispetto al sistema di riferimento oggetto e i tre angoli di rotazione $\omega, \varphi$ e $\kappa$ che individuano l'assetto della camera al momento della presa definiscono l'orientamento esterno (Gomarasca, 2004).

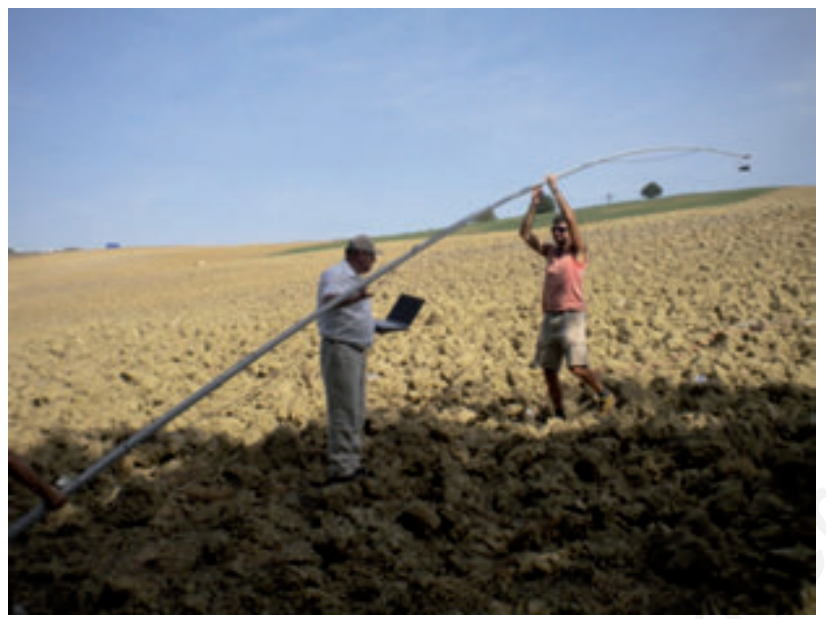

Figura 9. Momento in cui il palo viene esteso ed issato in posizione verticale.

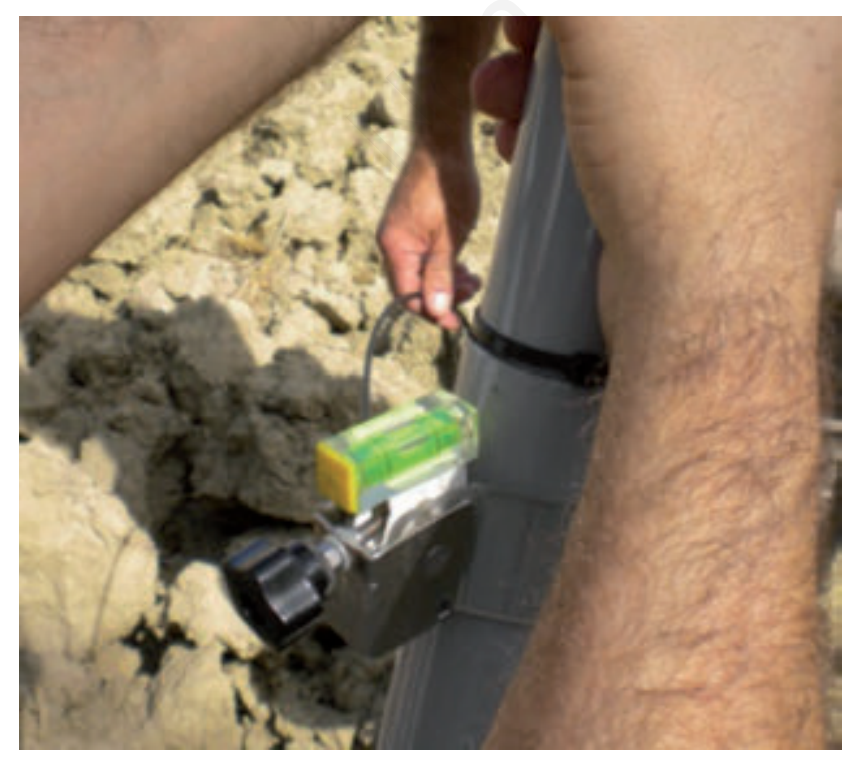

Figura 10. Messa in bolla del palo.
Questi parametri si compongono nelle equazioni di Collinearità e dimostrano come ad ogni punto oggetto corrisponda univocamente un punto immagine. Grazie a coppie di punti omologhi su stereo-coppie di foto è possibile risolvere tali equazioni e misurare le coordinate oggetto di ogni punto di coordinate immagine $\mathrm{x}, \mathrm{h}$.

Per il processo fotogrammetrico digitale è stato utilizzato il software ERDAS Imagine una suite completa di strumenti software specifici dedicati alle applicazioni del Telerilevamento.

In particolare per l'orientamento è stata utilizzata una suite di moduli software integrati (Leica Photogrammetry Suite-LPS) che fornisce strumenti per la fotogrammetria accurati ed una vasta gamma di applicazioni.

\section{Orientamento interno dei fotogrammi}

Le fasi iniziali di analisi delle immagini fino all'orientamento ester-

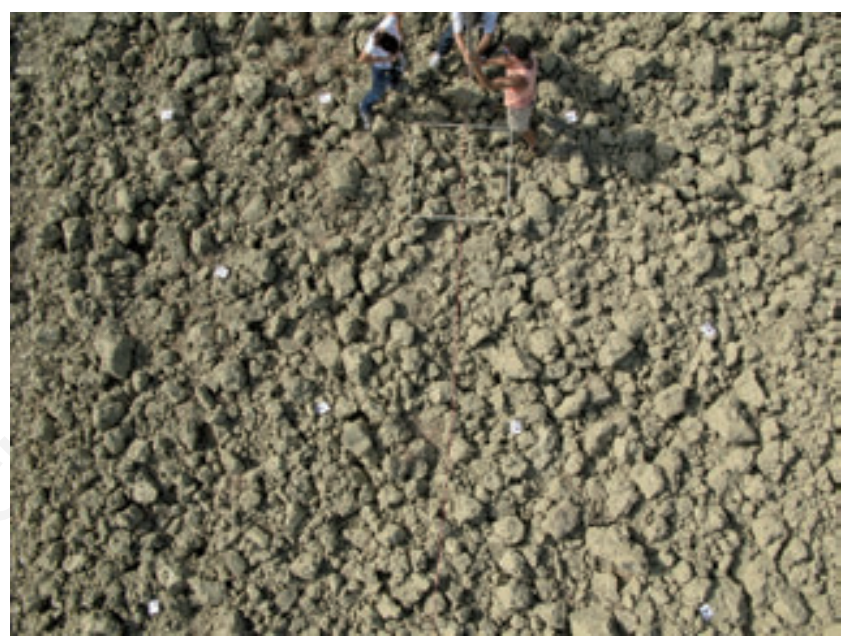

Figura 11. Foto zenitale scattata tramite la PAP.

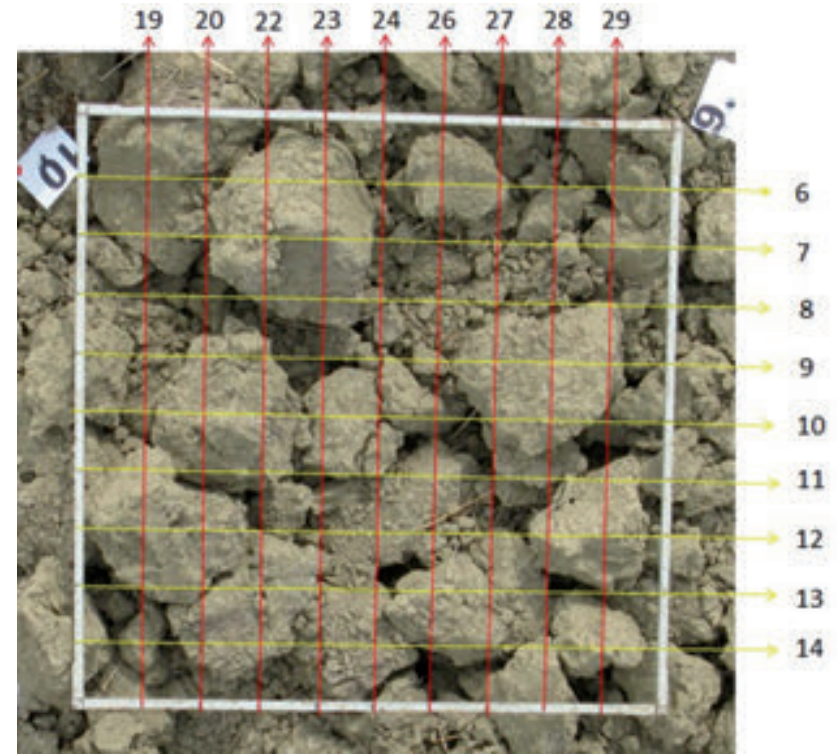

Figura 12. Griglia di rilievo dei profili. 
no sono state comuni ad entrambe le sessioni di rilievo e vengono qui trattate insieme.

L'orientamento interno è iniziato con la scelta delle immagini più appropriate che permettevano di avere un migliore overlap e maggiore costanza nella direzione della presa in maniera da mitigare gli errori geometrici. Scelte le foto, è stato preparato il block file (Figura 13). Il Blk è un file binario che contiene tutte le informazioni associate al progetto fotogrammetrico (sistema di riferimento, immagini scelte, informazioni sul sensore, GCP e i tie point, ecc). Dato un nome al blk è stato selezionato, come modello geometrico, la Digital Camera.

Assegnato il sistema di riferimento con cui sono stati rilevati i GCP (UTM-WGS84 fuso32), sono state inserite tutte le specifiche di scatto di cui si disponeva tra: sistemi di rotazione, direzione di scatto delle foto, distanza media dal suolo e parametri relativi all'orientamento esterno. Dato che si tratta di foto nadirali la direzione della foto è stata

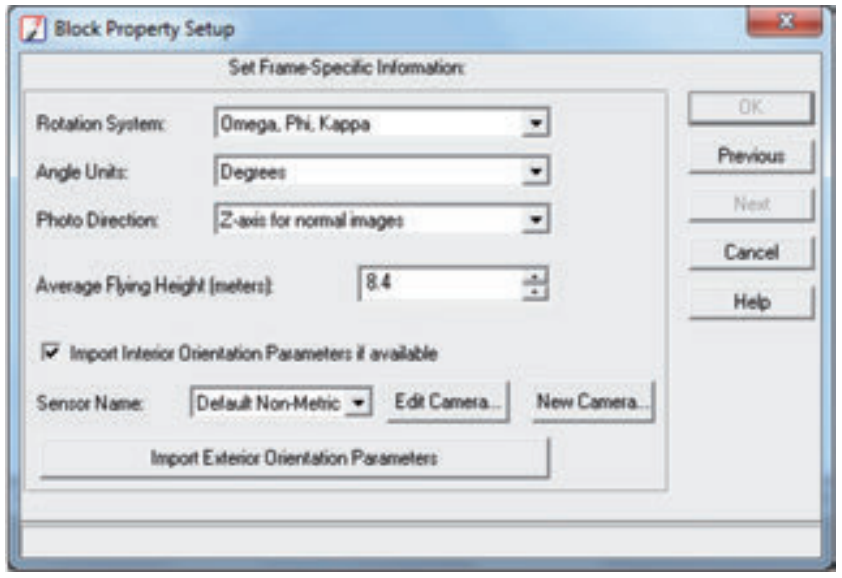

\begin{tabular}{|c|c|c|c|}
\hline \multicolumn{3}{|c|}{ = Proprietà - Capture_00028 } & E \\
\hline \multicolumn{4}{|c|}{ Sicurezza } \\
\hline Risutati scansione & Dettagl & \multicolumn{2}{|c|}{ Versioni precedent } \\
\hline Proprieta & \multicolumn{2}{|l|}{ Valore } & \multirow[t]{6}{*}{ a } \\
\hline Fotocamera - & & & \\
\hline Produttore fotocamera & \multicolumn{2}{|l|}{ Canon } & \\
\hline Modelo fotocamera & \multicolumn{2}{|c|}{ Canon PowerShot G7 } & \\
\hline Fetop & \multicolumn{2}{|l|}{$f / 4$} & \\
\hline Tempo di esposizione & \multicolumn{2}{|c|}{$1 / 500 \mathrm{sec}}$. & \\
\hline Sensibità ISO & & & D \\
\hline Esposizione & \multicolumn{2}{|c|}{ Opassaggio } & \\
\hline Distanza focale & \multicolumn{2}{|l|}{$7 \mathrm{~mm}$} & E \\
\hline Massima apertura & \multicolumn{2}{|l|}{296875} & \\
\hline
\end{tabular}

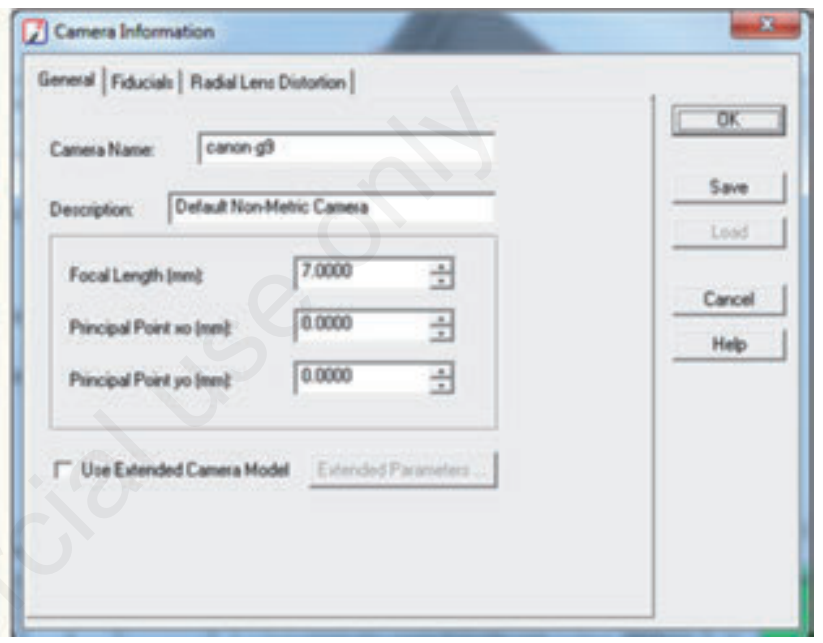

Figura 14. Proprietà di uno dei file delle foto scattate con la Pap e il tab Camera Information.

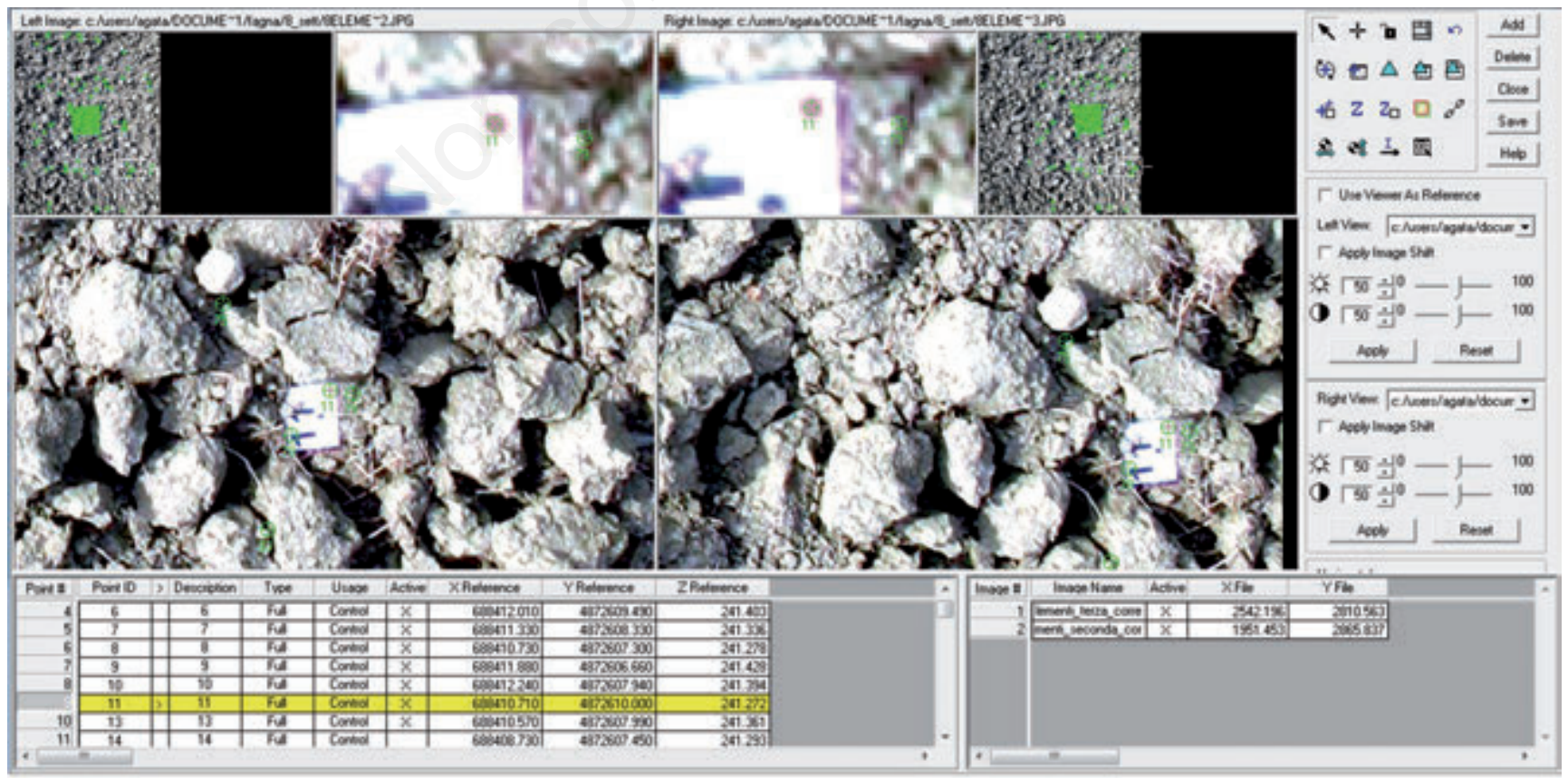

Figura 15. Point Measurement. 
lasciata $Z$ axis for normal images, ed è stata assegnata l'altezza media del volo (Figura 13). L'altezza del punto di presa è stata stimata attraverso appositi software grazie alle dimensioni note del frame. Infine è stato aggiunto il modello della camera selezionando New Camera. Nella camera information è stato specificato il nome della macchina fotografica e la distanza focale pari a $7 \mathrm{~mm}$ (come dalle proprietà del file immagine) (Figura 14). Tramite la funzione Add Frame sono state aggiunte le immagini precedentemente selezionate. Non essendo una camera metrica l'OI era limitato all'inserimento delle caratteristiche della camera. Attraverso il frame editor e la sottofinestra Interior orientation è stato impostato il pixel size relativo alla macchina fotografica utilizzata. Per trovare il pixel size, la ccd è stata divisa per il numero di pixel della foto. La CCD della canon g7 era di 1/1,8" che corrispondono a $7,176 \mathrm{~mm} \times 5,319 \mathrm{~mm}$. I pixel registrati per una dimensione immagine "L" erano pari a 3648px x 2736px facendo una semplice divisione è stato possibile ottenere la dimensione del pixel pari a $0,0019 \mathrm{~mm} / \mathrm{px} 0$ 1,9 micron. Con il tasto Next sono state rese attive le informazioni anche per le restanti immagini.

Una volta associati i fotogrammi al sistema meccanico che lo ha generato è stata creata una relazione tra i fotogrammi stessi ed il siste-

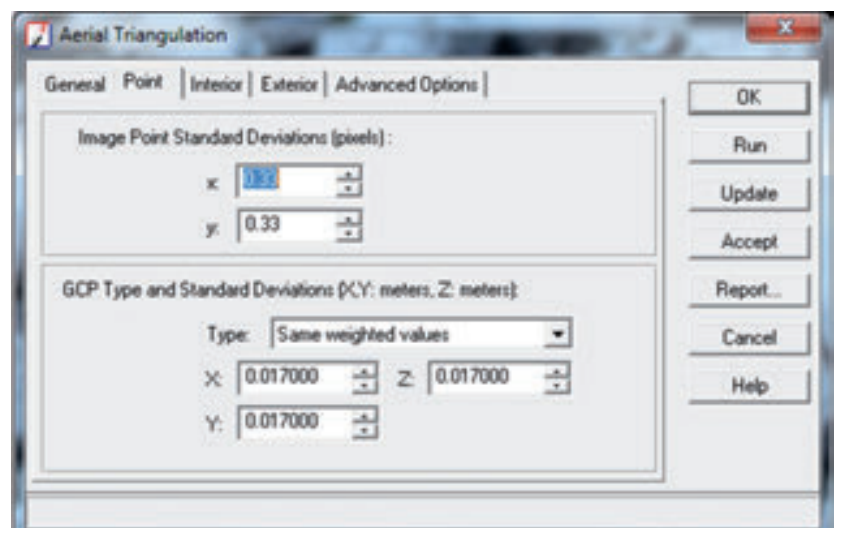

Figura 16. Proprietà di triangolazione.

\begin{tabular}{|c|c|c|c|c|}
\hline \multicolumn{2}{|c|}{ J Triangulation Summary } & & & $x$ \\
\hline \multirow{2}{*}{\multicolumn{3}{|c|}{ Triangulation Iteration Convergence. }} & Yes & \\
\hline & & 3522 & Close \\
\hline \multicolumn{2}{|c|}{ Control Poind RMSE: } & \multicolumn{2}{|c|}{ Check Point PMSE: } & Update \\
\hline \multirow{3}{*}{$\begin{array}{l}\text { Ground } X \\
\text { Ground } Y \text { : }\end{array}$} & \multirow{2}{*}{$0.0186(12)$} & \multirow{3}{*}{$\begin{array}{l}\text { Ground X } \\
\text { Ground } Y\end{array}$} & \multirow{2}{*}{$0.0000(0)$} & \multirow{3}{*}{ Report.. } \\
\hline & & & & \\
\hline & $0.0365[12]$ & & $0.0000(0)$ & \\
\hline Ground Z & $0.0136(12)$ & Ground $Z$ & $0.0000(0)$ & Review... \\
\hline Image $X$ & $0.0174(24)$ & Image X & $0.0000(0)$ & \multirow[t]{2}{*}{ Help } \\
\hline Image $Y$ : & $0.2837[24]$ & Image $Y$ : & $0.0000(0)$ & \\
\hline \multicolumn{2}{|c|}{ RMSE Significank Digts: } & 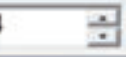 & & \\
\hline
\end{tabular}

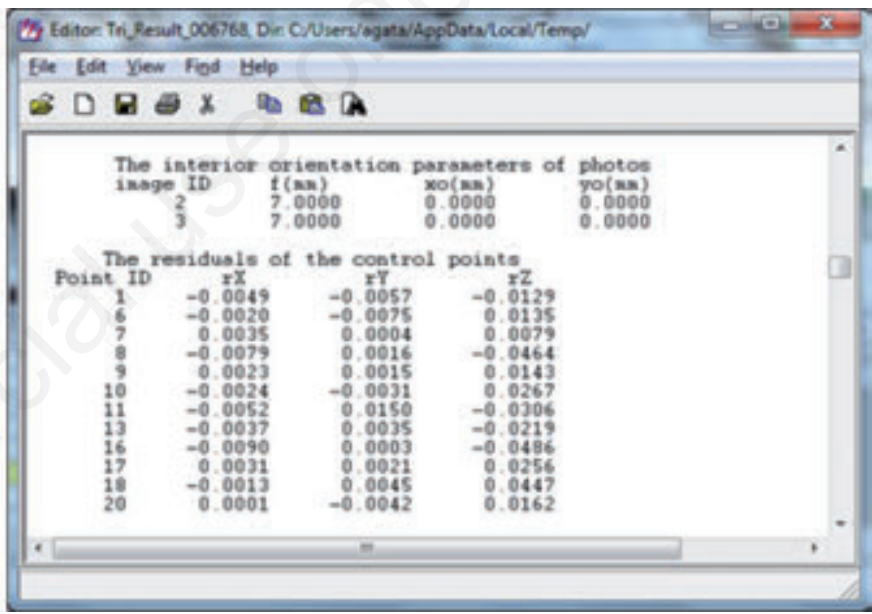

Figura 17. Triangulation Summary della prima sessione con relativo Report.

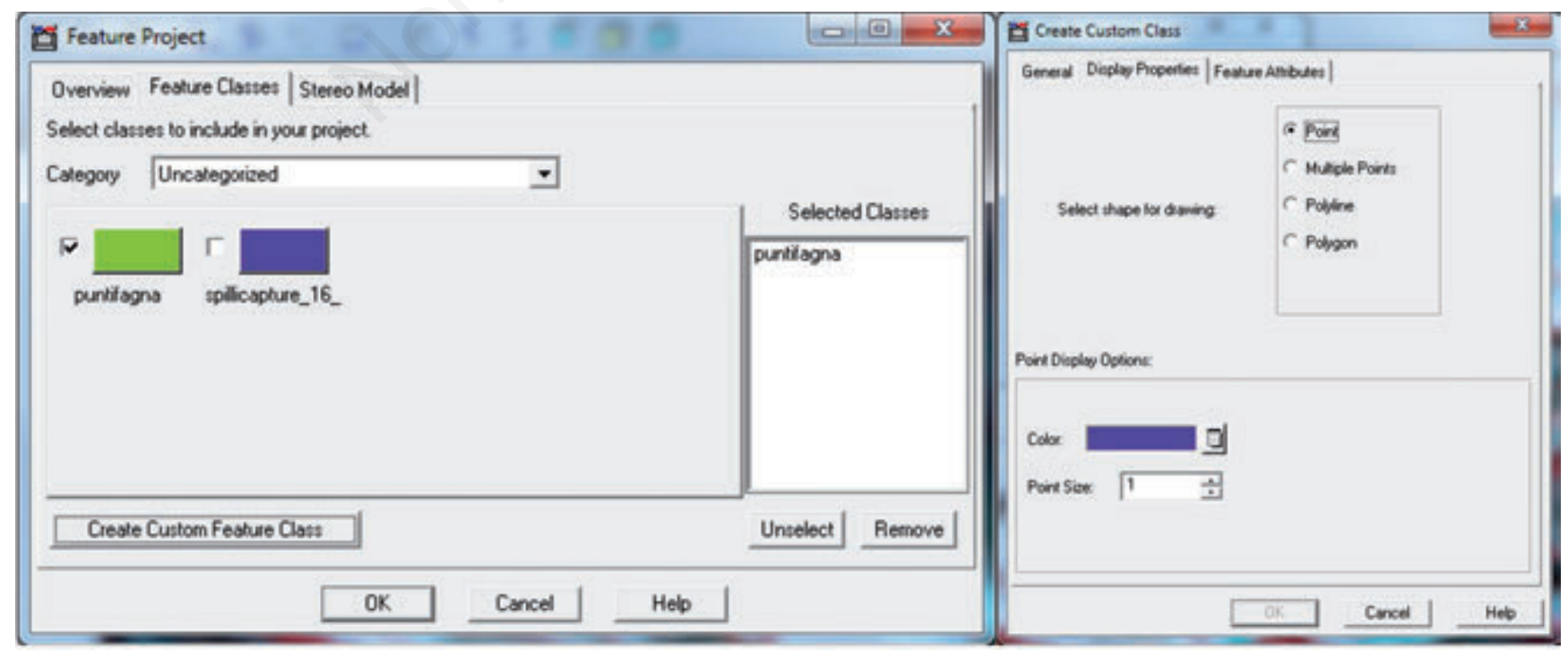

Figura 18. Finestre per la creazione di nuovi progetti e di nuove feature class. 
ma di riferimento terreno andando a definire l'orientamento esterno, che viene suddiviso in tre fasi:

- Riconoscimento e posizionamento sui fotogrammi dei punti di appoggio GCP(ground controll points)

- generazione automatica di Tie Points

- la triangolazione

L'inserimento dei punti di appoggio è stata effettuata con il Point Measurement (Figura 15). Ogni punto omologo è stato riportato su tutte le foto su cui era presente, introducendo le coordinate terreno del punto e assegnando le coordinate immagine sulle foto.

Dopo aver inserito tutti i GCP è stato avviato il processo di generazione automatica dei Tie points, provvedendo poi ad un loro infittimento manuale nelle aree di interesse. Terminato l'inserimento dei punti di appoggio e dei punti di legame, LPS aveva tutte le informazioni necessarie per eseguire la triangolazione aerea. Da Triangulation Properties è stato selezionato il tab Point all'interno del quale è stato possibile assegnare i pesi statistici ai GCP. Tali pesi sono comunemente definiti dall'accuratezza della posizione dei punti calcolata durante il

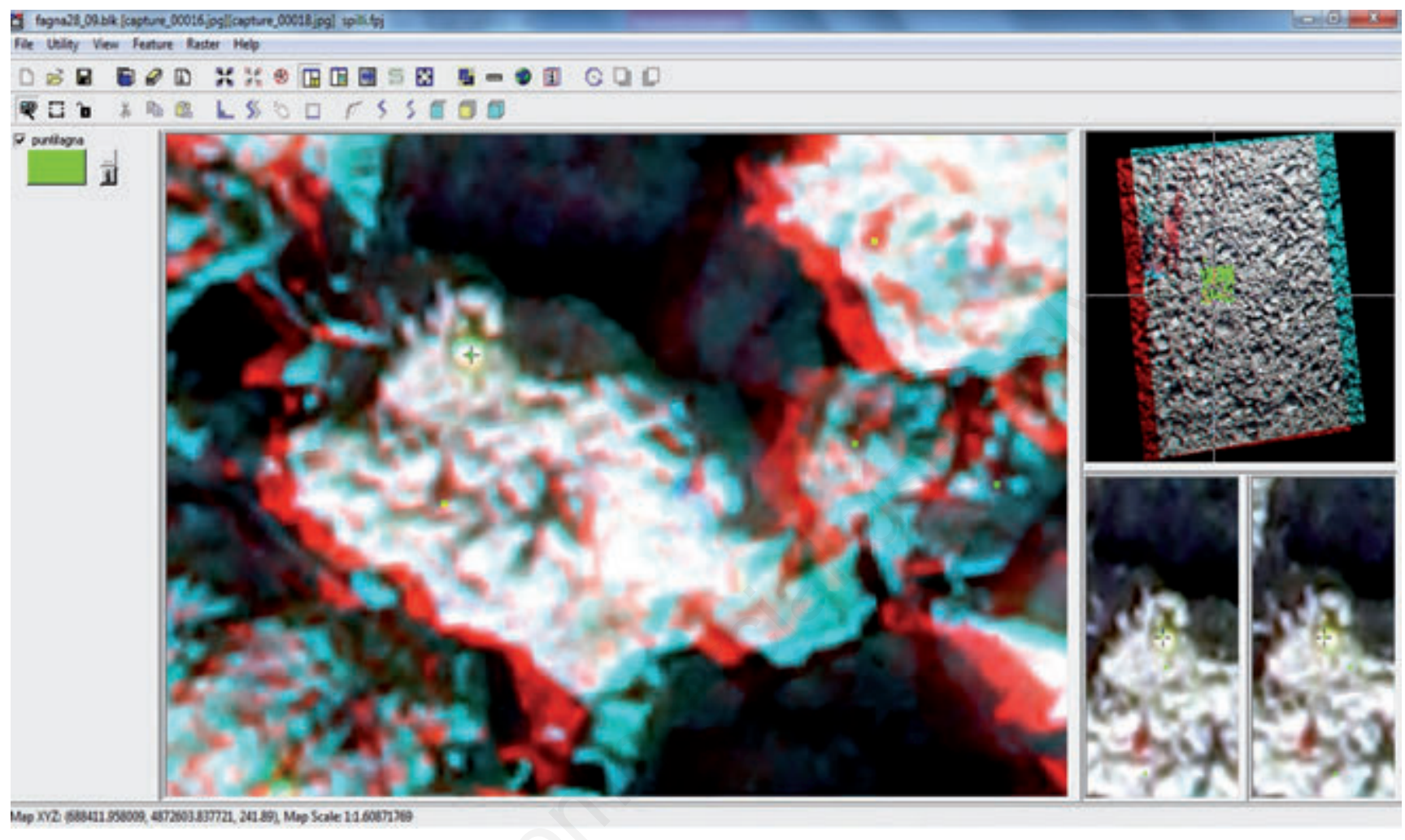

Figura 19. Stereo Analist Feature Project.

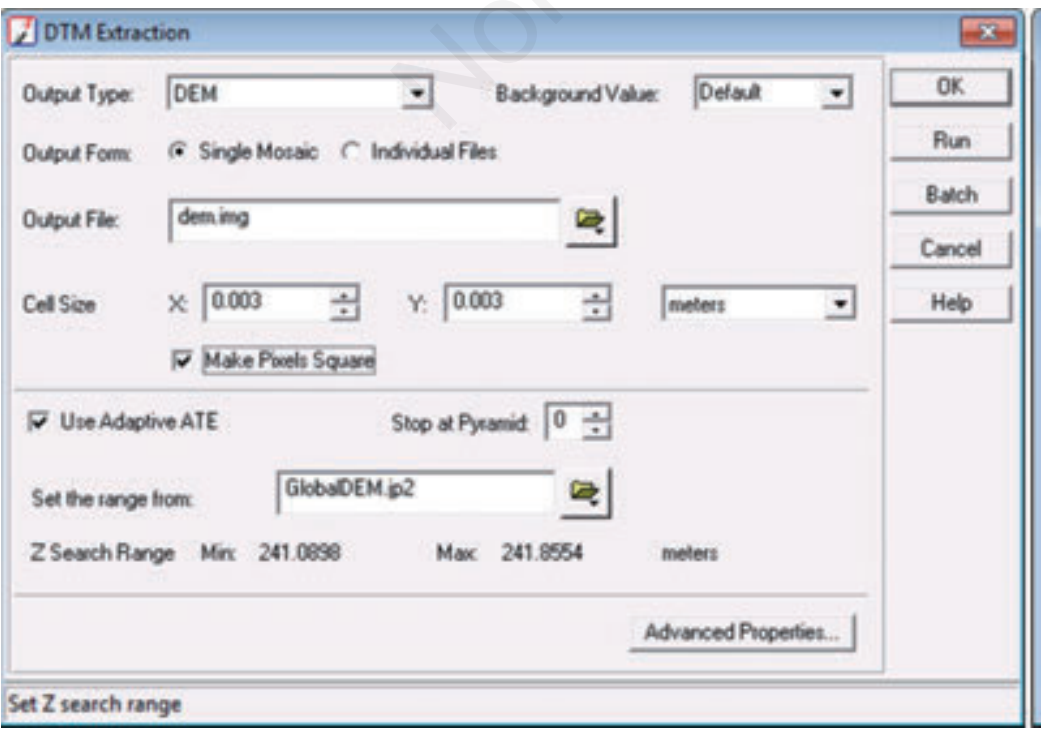

\begin{tabular}{|c|c|c|c|c|}
\hline \multicolumn{5}{|c|}{ 2] DTM Extraction Properties } \\
\hline General | Image Pait | & \multicolumn{2}{|c|}{ | Area Selection | Accurscy | } & \multicolumn{2}{|c|}{ Seed Data | } \\
\hline Horizontal Phoiection & \multicolumn{2}{|l|}{ UTM } & \multirow{5}{*}{\multicolumn{2}{|c|}{ Set.. }} \\
\hline Horizontal Sphercid & \multicolumn{2}{|l|}{ WGS 84} & & \\
\hline Zone Number: & \multicolumn{2}{|l|}{32} & & \\
\hline Herizontal Dahum. & \multicolumn{2}{|l|}{ WGS 84} & & \\
\hline Horizental Units: & metert & 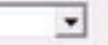 & & \\
\hline Vestical Spheroid. & \multicolumn{2}{|l|}{ WGS 84} & \multirow{3}{*}{\multicolumn{2}{|c|}{ Set. }} \\
\hline Vertical Dotum & \multicolumn{2}{|l|}{ WaS 84} & & \\
\hline Veftical Units: & meters & 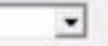 & & \\
\hline \multicolumn{5}{|c|}{ F Create DTM Poink Status Output Imsge } \\
\hline \multicolumn{3}{|c|}{ F Reduce DTM Coenelation Asea by } & 108 & \pm \\
\hline \multicolumn{3}{|c|}{ F Tim the DTM Boeder by } & $5 \%$ & $\div$ \\
\hline
\end{tabular}

Figura 20. DTM Extraction ed Advanced Properties. 
post-processing ed inserita in GCP standard deviation scegliendo nel flag type la voce Same Weightet Values (Figura 16). Dopo averne settato i parametri è stata eseguita la triangolazione e ne è stato valutato l'errore totale in pixel come scarto quadratico medio. Per verificarne meglio i risultati è stato visualizzato il Report di dettaglio, accessibile dalla finestra Triangulation Summary, nel quale sono riportati gli errori su X, Y e Z di ogni singolo punto di controllo. Questo è stato utile per verificare se tra i GCP considerati ce ne fossero alcuni con errori molto elevati, consentendo una loro eventuale correzione o esclusione dal calcolo (Figura 17).

Dopo le valutazioni e correzioni effettuate, le triangolazioni di entrambe le sessioni di rilievo hanno dato esito positivo con uno scarto molto contenuto. A questo punto le due sessioni hanno seguito strade diverse.

\section{Prima sessione: estrazione delle quote tramite restituzione stereoscopica}

Lo scopo della prima sessione di rilievo è stato quello di determinare l'accuratezza raggiunta dalle quote dei punti restituiti con ERDAS. Per far ciò è stata utilizzata la suite di ERDAS Stereo Analyst che contiene una ricca collezione di funzioni per il 3D e le applicazioni per l'editing stereoscopico.

La modalità utilizzata per la stereovisione è stata l'anaglifa con combinazioni di colori Red e Green+Blue(cyan) (Figura 18). Questa modalità è sicuramente la più semplice e meno costosa tra le tecniche di

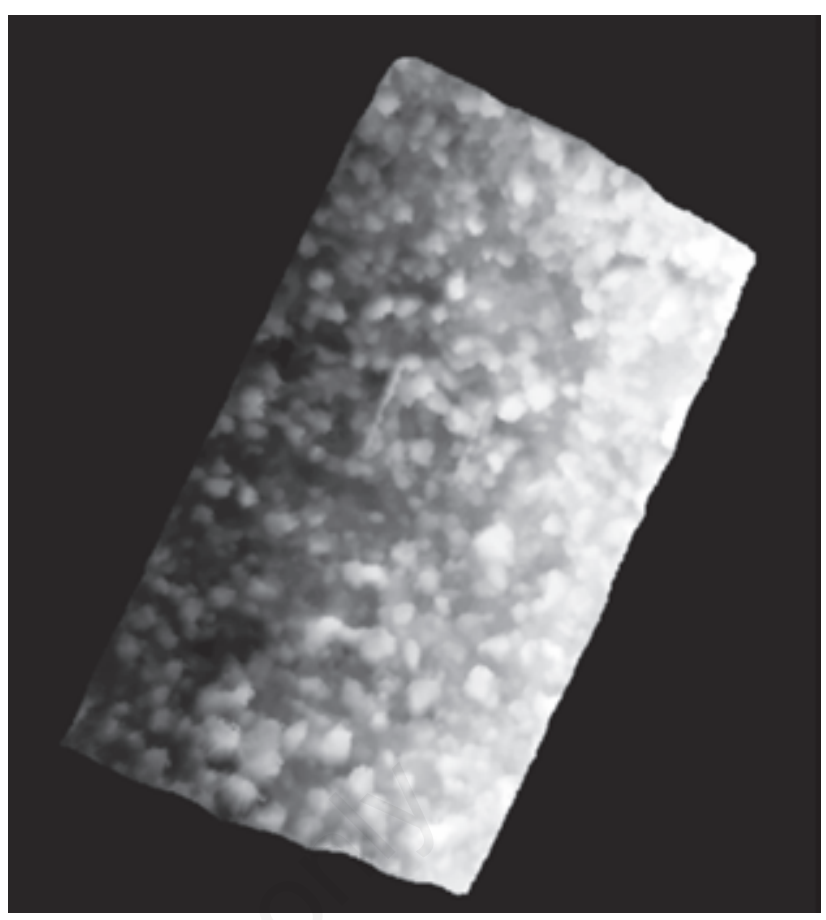

Figura 21. DEM estratto dal software ERDAS.

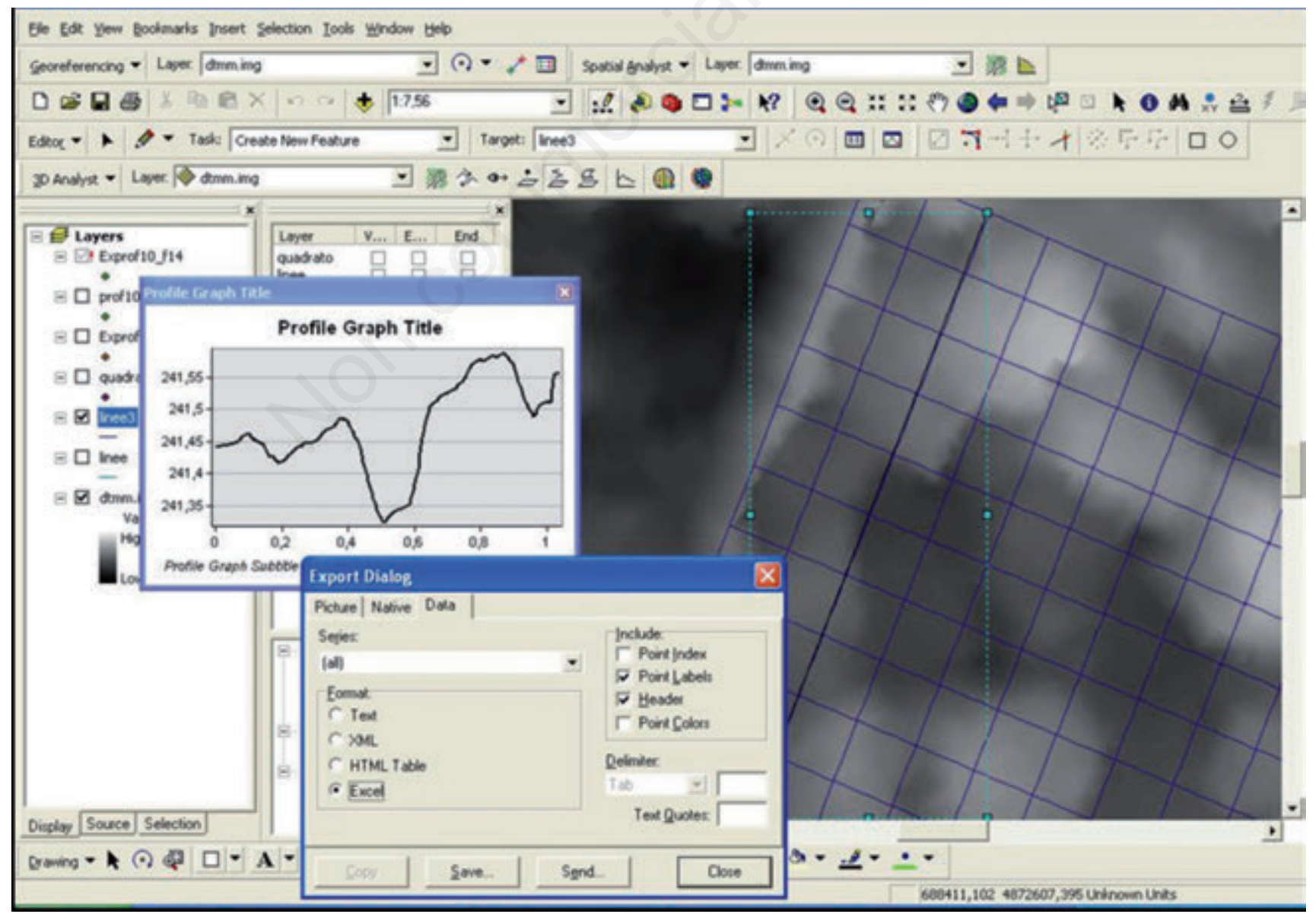

Figura 22. Estrazione del profilo con 3D Analist. 
restituzione, realizzabile con un semplice monitor di computer e con l'uso degli appositi occhiali con lenti a due diversi colori, rosso e blu. Tramite Stereo Analist è stato creato un nuovo progetto a cui è stata associata tramite Create Custom Class una nuova feature class chiamata puntifagna, alla quale è stata data forma puntuale, colore verde e come attributi la quota e la label di riconoscimento.

Dal tab Stereo Model, aprendo il blk della prima sessione, è stata scelta la stereo-coppia di foto. A questo punto è stato aperto il Workspace di Stereo Analist che si presentava come in Figura 19, riportando sulla sinistra la feature class puntifagna. Nella Main view, aumentando lo zoom-in, il cursore veniva spostato nei pressi delle puntine, facendo poi scivolare le due immagini l'una sull'altra veniva posizionato alla quota desiderata dove cliccando con la feature class puntuale attiva veniva editato il punto. A causa del colore delle puntine si è rivelato particolarmente difficile individuarle in modalità anaglifa, per cui sono state restituite solo parte di esse. La feature generata dalla restituzione è stata poi esportata in formato 3Dshape la cui tabella è stata esportata in excel, per metterla a confronto con i punti rilevati con il GPS.

\section{Seconda sessione: estrazione del DEM e dei profili}

La seconda sessione di rilevo prevedeva l'estrazione del dem, ed il confronto dei profili ottenuti da questo in ambiente Gis, con quelli rilevati in campo tramite profilimetro ad aghi.

Riprendendo con LPS il blk della seconda sessione contenente tutti i parametri della triangolazione, è stata avviata l'estrazione automatica del DEM, mediante la funzione DTM Extraction (Figura 20). Qui è stato scelto come output un DEM che utilizzasse per l'estrazione tutte le coppie di immagini attive (single mosaic), con una dimensione di pixel pari a quello dell'immagine cioè $3 \mathrm{~mm}$ x $3 \mathrm{~mm}$. Nelle proprietà avanzate sono stati attivati i tre flag del tab General: trim the DTM border per tagliare il bordo del DEM di un 5\% (escludendo così una zona spesso caratterizzata da bassa accuratezza), Reduce DTM Correlation Area per ridurre l'area di correlazione delle due coppie di immagini di un $10 \%$ in modo che la parte esterna del modello non venisse inclusa nell'estrazione ed infine il flag per creare un file raster che illustrasse la qualità associata al DEM in termini di correlazione. Il DEM estratto con ERDAS (Figura 21) è stato poi elaborato con Arc-Gis. Sul raster dem sono stati caricati i punti rilevati sugli spigoli del frame ed è stata ricostruita la griglia di rilievo. Il posizionamento del frame è stato poi regolato tenendo conto dello scarto quadratico medio dei punti GPS, e della sua sagoma nettamente visibile sul dem stesso. Grazie a 3danalist e alla sua funzione create profile graph sono stati estratti i grafici e successivamente esportati in formato data in excel.

Completata l'estrazione dei 18 profili dal DEM (Figura 22), sono stati digitalizzati i profili ricavati con profilimetro ad aghi. Prima operazione eseguita è stata l'orto-rettifica delle foto, per renderle perpendicolari al punto di presa. Tale operazione è stata possibile grazie alla conoscenza delle misure precise del profilimetro e al Georeferencing di Arc-Map utilizzando un sistema di riferimento fittizio in metri. Inserite le coordinate (Figura 23), tramite la trasformazione Adjust le foto sono state rettificate. Creata una feature puntuale con attributi X,Y e Z, sono state digitalizzate le estremità superiori degli aghi (Figure $24 \mathrm{e} 25$ ). Le tabelle di ogni profilo ottenuto sono poi state esportate in excel. Ottenuti i

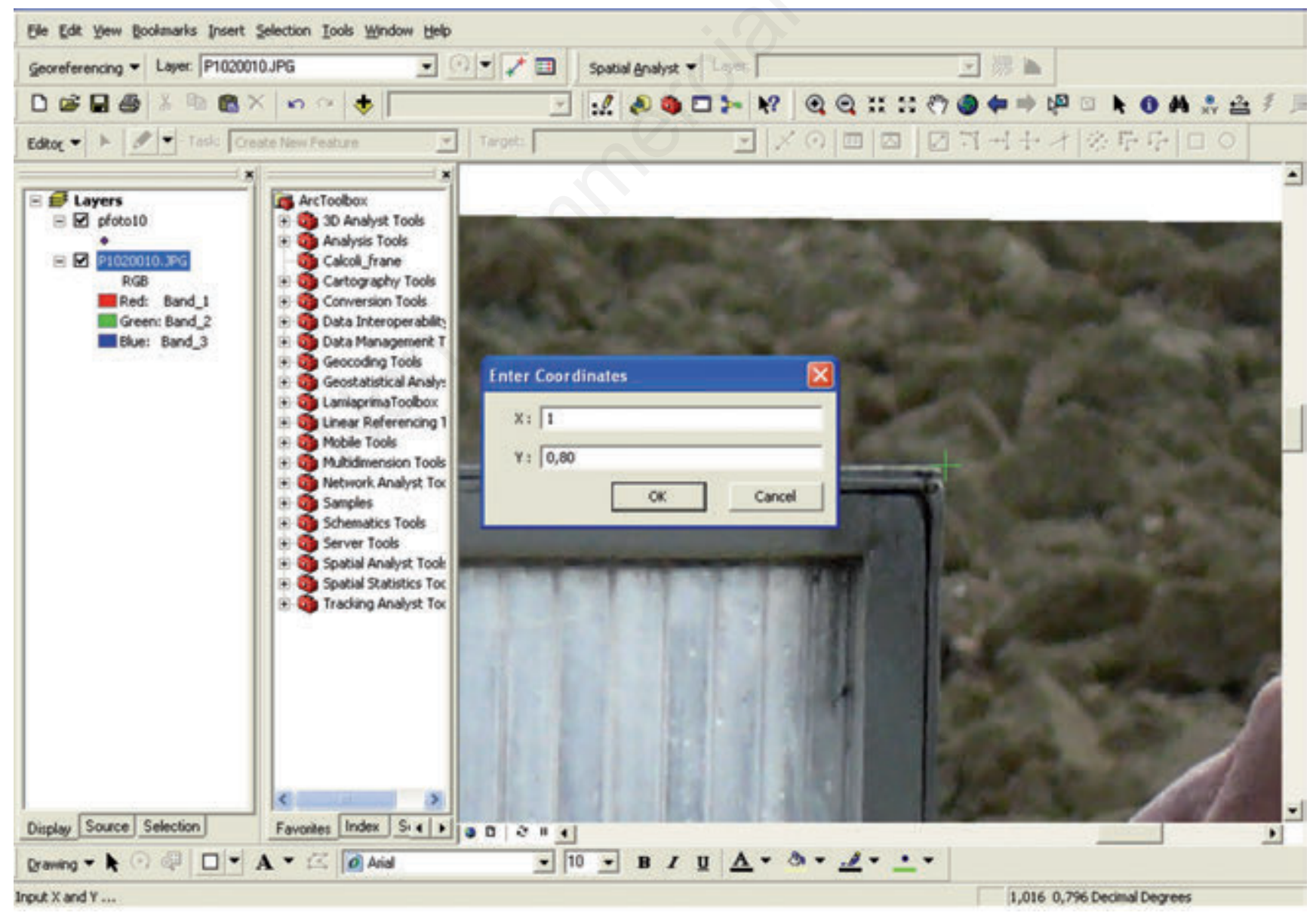

Figura 23. Attribuzione delle coordinate agli spigoli del frame del profiligrafo. 
dati digitali dei profili di entrambi i metodi di rilievo, è stato possibile preparare i file con estensione dat necessari per eseguire il software di analisi della rugosià Mric.

Il Software Multiple Roughness Index Computation sviluppato nel CNR-IGES da Borselli permette di calcolare rapidamente una serie di indici di rugosità da dati $2 \mathrm{~d}$ tra i quali la Random Roughness (RR) e l'indice di Tortuosità (T).

\section{L'indice RR (Random Roughness)}

RR (random roughness) è il più noto indice di rugosità, nella formulazione definitiva di Currence e Lovely (1970) è espresso come la deviazione standard delle altezze relative del profilo o della superficie campionate secondo un passo o maglia regolare:

$$
\mathrm{RR}=\sqrt{\sum_{\underline{i}=1}^{n}\left(Y_{i}-\mu_{y}\right) / n}
$$

dove: $n$ è il numero delle altezze relative campionate; $\mu_{y}$, la media aritmetica delle altezze campionate; $Y_{i}$ il valore di ogni singola altezza relativa nel profilo (Figura 26).
L'indice RR (dimensionalmente una lunghezza, L) è estremamente facile da calcolare; tuttavia, è necessario procedere ad una preliminare eliminazione di trend lineari o non lineari eventualmente presenti sull'intero profilo e determinati, ad esempio, dalla pendenza locale. In questo caso, l'indice RR viene calcolato sui residui rispetto al trend rimosso. L'operazione di rimozione del trend, operata normalmente mediante una regressione lineare o polinomiale, è estremamente importante per filtrare la rugosità casuale in profili raccolti lungo una componente di pendenza principale 0 per calcolare la rugosità sovrimposta su rugosità di ordine superiore. L'indice RR è il più utilizzato tra quelli esistenti, grazie alla facilità di calcolo e all'esistenza di una serie di relazioni empiriche che lo legano ad alcune proprietà fisiche della superficie, quali lo storage idrico superficiale (Onstad, 1984; Kamphorst et al., 2005) e la resistenza idraulica (Gilley e Finkner, 1991).

\section{L'indice T (tortuosità)}

L'indice di tortuosità (T) è adimensionale e rappresenta il rapporto fra la lunghezza totale (Leff) dello sviluppo di una sezione (transetto) superficiale del suolo e la sua proiezione (Lh) perpendicolare sul piano; T= Leff/Lh (Boiffin, (1984) (Figura 27). Per maggiori dettagli sulla modalità di calcolo vedi Borselli (1998).

In un file txt i dati sono stati sistemati in due colonne: la prima contenente le coordinate $\mathrm{x}$ ad intervalli costanti delle misurazioni di quota

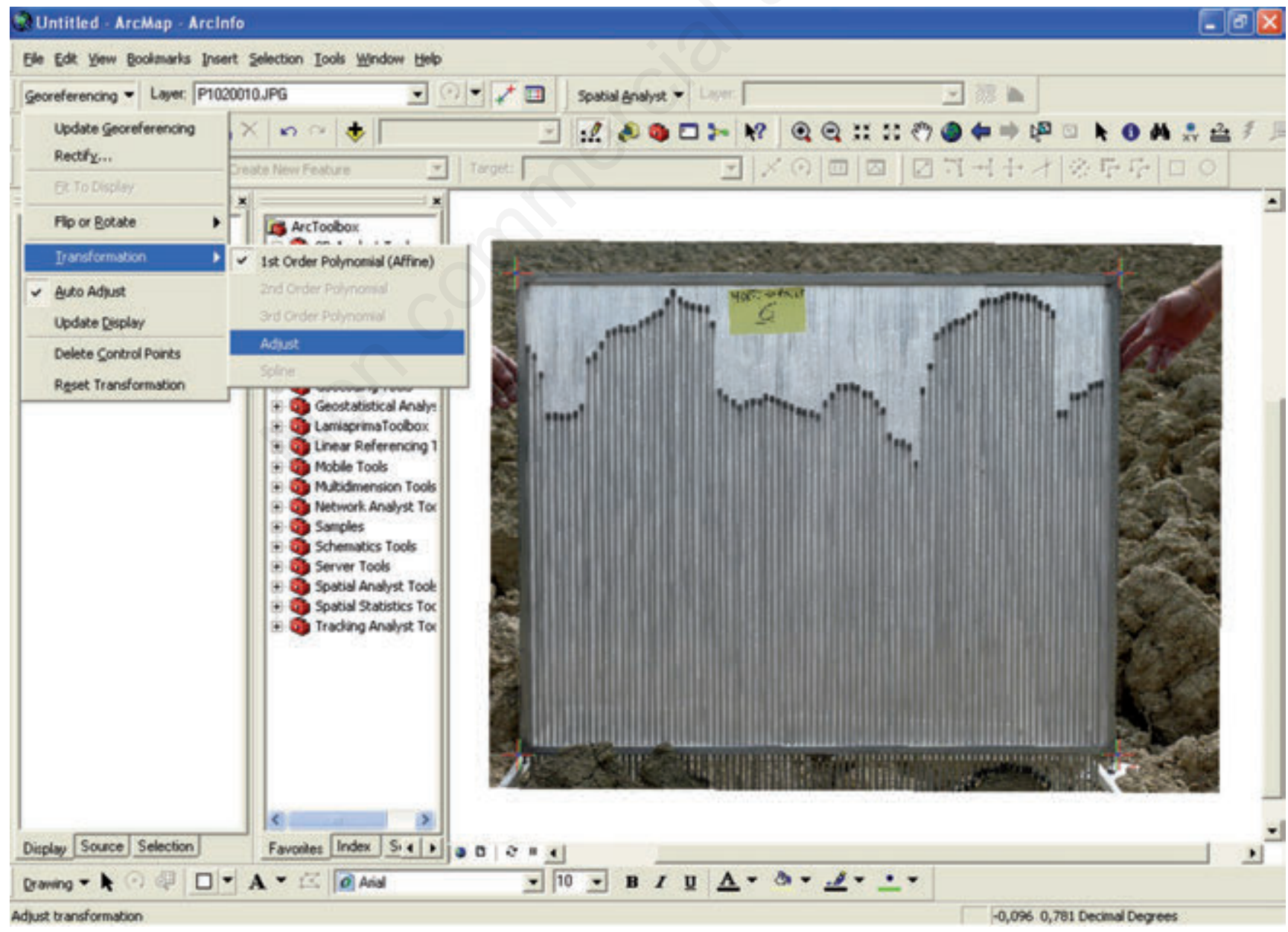

Figura 24. Digitalizzazione delle estremità superiori degli aghi del profiligrafo. 
effettuate, la seconda colonna il valore della quota corrispondente (i dati sono in centimetri). Il programma può analizzare in un'unica battuta una lista di file, ognuno rappresentante un profilo differente di suolo. Il risultato dell'analisi è un Ascii text file, nel quale per ogni file di profilo vengono restituiti i valori degli indici di rugosità.

\section{Risultati}

\section{Prima sessione di rilevo}

Dalla prima sessione di rilievo sono stati ricavati due set di dati uno realmente misurato in campo e uno misurato con software ERDAS e le sue suite.

Dei 100 punti rilevati con il GPS, 68 sono stati riconosciuti a video tramite Stereoanalist.

Nella Tabella 2 sono state riportate le statistiche descrittive delle due serie di dati. Tutti valori degli statistici descrittivi si sono rivelati molto vicini in particolare per i valori medi e massimi. Per i valori minimi si notano i maggiori scostamenti. Il test $t$ (Tabella 3 ) mostra una differenza significativa fra medie (Quote GPS meno Quote PAP-ERDAS) pari a $-1,9 \mathrm{~cm}$. Ciò significa che la metodologia PAP-ERDAS, nelle condizioni
Tabella 2. Statistiche descrittive delle due serie di dati GPS e ERDAS.

\begin{tabular}{lcc} 
Statistica descrittiva & Dati GPS & Dati BRDAS \\
Media & 241,6284 & 241,6482 \\
Errore standard & 0,0087 & 0,0075 \\
\hline Mediana & 241,6342 & 241,6500 \\
Moda & 241,6646 & 241,6300 \\
\hline Deviazione standard & 0,0716 & 0,0615 \\
Varianza campionaria & 0,0051 & 0,0038 \\
\hline Curtosi & $-0,5834$ & $-0,6091$ \\
Asimmetria & $-0,0571$ & $-0,0352$ \\
\hline Itervallo & 0,3065 & 0,2500 \\
Minimo & 241,4779 & 241,5300 \\
\hline Massimo & 241,7844 & 241,7800 \\
Somma & 16430,7334 & 16432,0800 \\
\hline Conteggio & 68 & 68 \\
Più grande (1) & 241,7844 & 241,7800 \\
\hline Più piccolo (1) & 241,4779 & 241,5300 \\
Livello di confidenza (95\%) & 0,0173 & 0,0149
\end{tabular}

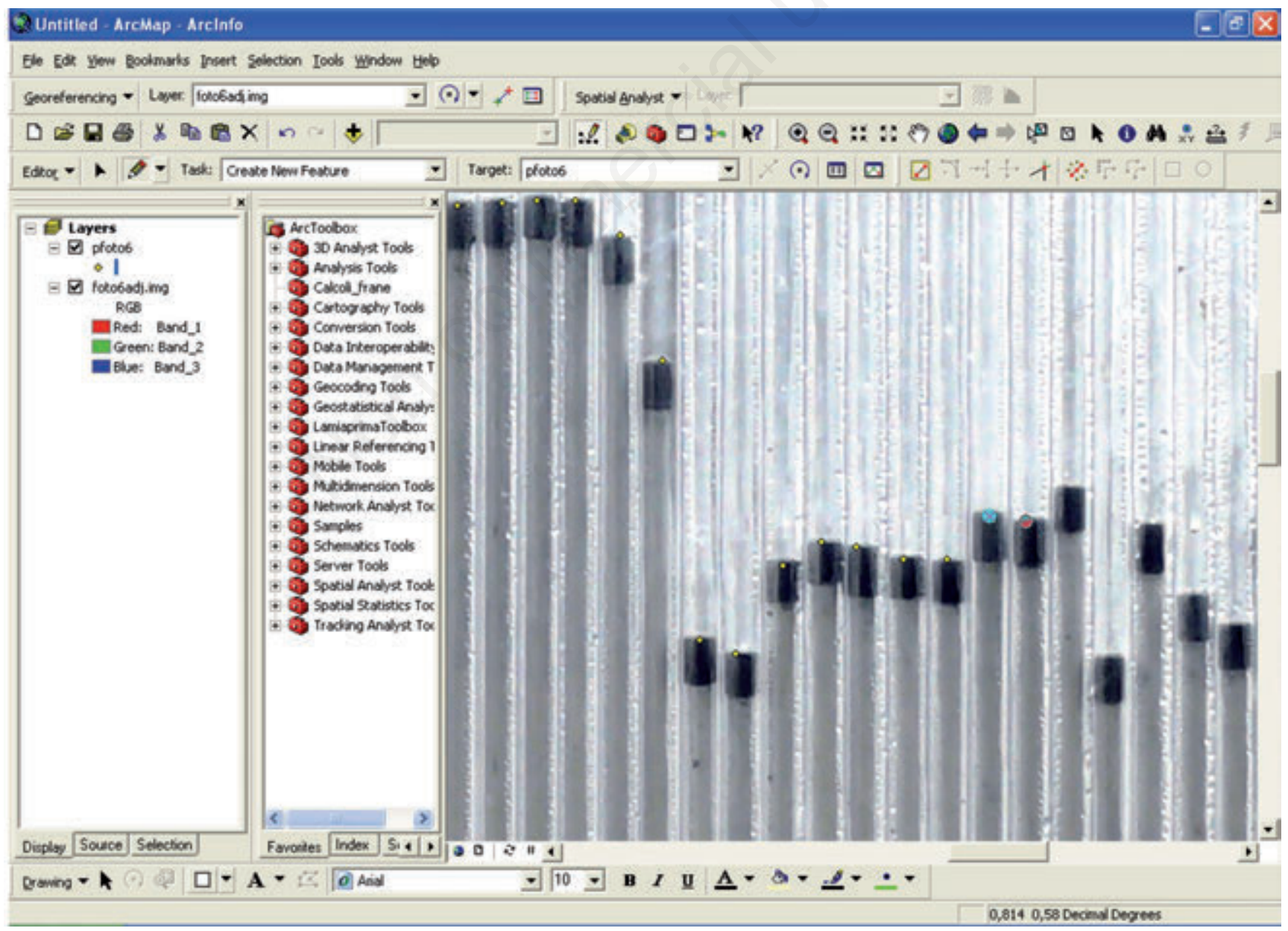

Figura 25. Particolare della digitalizzazione delle estremità superiori degli aghi del profiligrafo. 
di suolo indagate (cioè con grosse zolle), pone le quote ad un valore mediamente più alto, per la difficoltà di discriminare le zone fortemente in ombra (avvallamenti).

\section{Seconda sessione di rilievo}

Scopo della seconda sessione era quello di confrontare la rugosità stimata attraverso metodi classici come i profilimetri e quella ricavata tramite l'analisi del DEM.

Dai profili estratti sono stati generati grafici che hanno fornito una prima informazione sulla qualità della stima. È risultata evidente la tendenza da parte di ERDAS a smussare maggiormente le linee, in particolare per i valori bassi riguardanti gli avvallamenti del terreno. Un'altra evidente deformazione osservabile in Figura 28 (grafico di destra) è la risalita finale dovuta alla presenza del frame che si è rivelato un elemento di disturbo per l'interpolazione. I dati alla base dei grafici sono stati poi esaminati tramite il software MRIC. Fra gli indici ricavati in automatico vengono riportati in Tabella 4 solo l'indice RR e l'indice T. Confrontando i valori medi con il test $t$ di Student (Tabella 5) si rileva che non c'è differenza significativa fra medie per l'indice $\mathrm{RR}$, ricavato con le due metodologie. Riguardo all'indice di Tortuosità $\mathrm{T}$ il confronto fra medie mostra una differenza significativa, anche se di entità modesta (Tabella 6). I profili che hanno presentato maggiori differenze nei valori dell'indice sono quelli identificati con i numeri 13 , 29, 22, 20 in Tabella 4. Le stereofoto relative a profili presentano tutte zone di ombra che il software non è riuscito ad elaborare e contemporaneamente presentano l'interferenza del frame sui lati.

\section{Discussione critica dei risultati}

\section{La prima sessione di rilevo}

Per quanto riguarda la prima sessione di rilevo si può affermare che la metodologia PAP fornisce stereo-coppie di foto dalle quali è possibile raggiungere tramite restituzione un'accuratezza elevata sulle quote dei punti di interesse. Occorre però considerare che la scelta dei punti di controllo non è stata ottimale, non tanto per la loro forma (puntine da disegno) ma per il colore, la scelta dovrebbe ricadere su combinazioni di colori radiometricamente più contrastanti, in maniera da essere facilmente individuabili in modalità anaglifa.

\section{La seconda sessione di rilievo}

Per quanto riguarda la seconda sessione di rilevo si può affermare che la metodologia PAP permette di ricavare un DEM che consente di ottenere una buona stima della rugosità.

Nella seconda sessione si sono manifestati alcuni aspetti della metodologia utilizzata che possono essere migliorati e sviluppati. Innanzi tutto la scelta dell'avanzamento del palo sulla linea di volo (Figura 29A) si è rivelata poco adatta per via dell'interferenza causata dalla presenza nelle immagini (fascia rossa) della base del palo stesso e degli operatori. Ciò ha comportato una limitazione nell'estrazione del DEM dovendo considerare solo le porzioni comuni delle immagini non contenenti tali elementi. Un avanzamento laterale del palo non sulla linea di volo ma su una linea parallela consentirebbe un utilizzo migliore delle foto ottenute con la PAP (Figura 29B). Inoltre, per limitare il contrasto delle ombre che è causa dei maggiori scostamenti nei minimi degli statistici descrittivi, potrebbe essere utile effettuare i rilievi in condizioni di luce più morbida, ossia in una giornata con cielo nuvoloso. Ciò potrebbe consentire di avere una maggiore penetrabilità dei raggi di convergenza negli avvallamenti permettendo una stima migliore degli stessi.

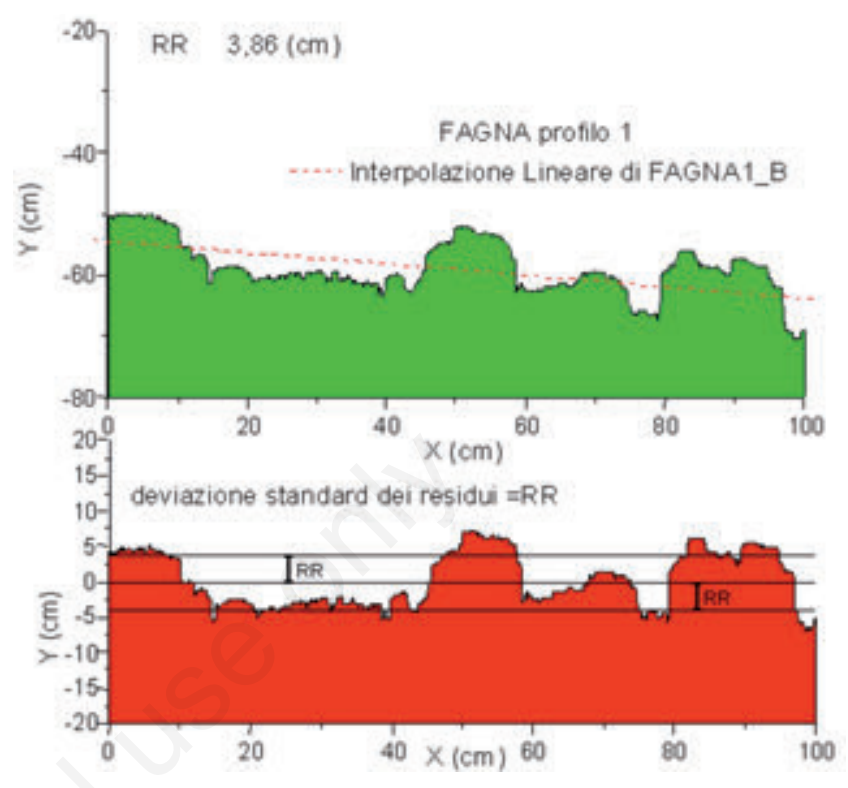

Figura 26. Indice RR. Esemplificazione della modalità di calcolo di RR e dell'eliminazione del trend dei profili di rugosità, importante per eliminare (o calcolare ) l'effetto pendenza. Il trend dei profili di rugosità viene normalmente rimosso prima di fare il calcolo degli indici di rugosità.

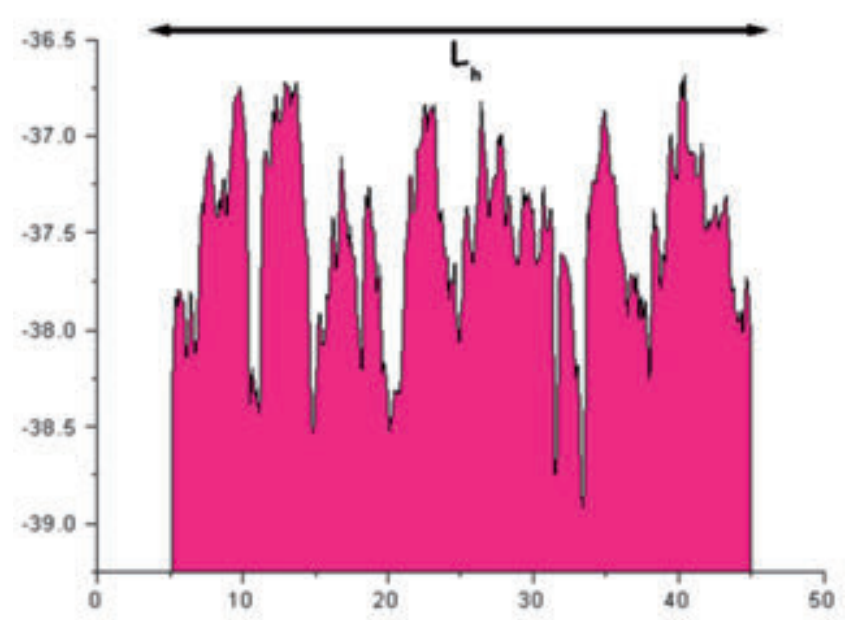

Figura 27. Indice T, esemplificazione.

Tabella 3. Test $t$ fra le quote medie rilevate con GPS e quelle rilevate con metodologia PAP.

\begin{tabular}{|c|c|c|c|c|c|c|c|c|}
\hline \multirow{2}{*}{ Test t per campioni dipendenti } & \multicolumn{7}{|c|}{ Diff. marcate significative al livello $\mathrm{P}<0,05000$} & \multirow[b]{2}{*}{$\mathbf{P}$} \\
\hline & Media & Dv.Std. & $\mathbf{N}$ & Diff. & Dv.Std. Diff. & $\mathrm{t}$ & gl & \\
\hline ZGPS & 241,6284 & 0,071602 & & & & & & \\
\hline Z ERDAS & 241,6482 & 0,061545 & 68 & $-0,019803$ & 0,026428 & $-6,17886$ & 67 & 0,000000 \\
\hline
\end{tabular}


L'interferenza invece mostrata dal Frame può essere migliorata applicando il comando Excluded area al momento dell'estrazione, tagliandolo ed estrapolando un DEM della sola area interna al Frame. Non particolarmente agevole è risultato poi per entrambi i rilievi il mantenimento in bolla del palo e la completa eliminazione dell'oscilla-

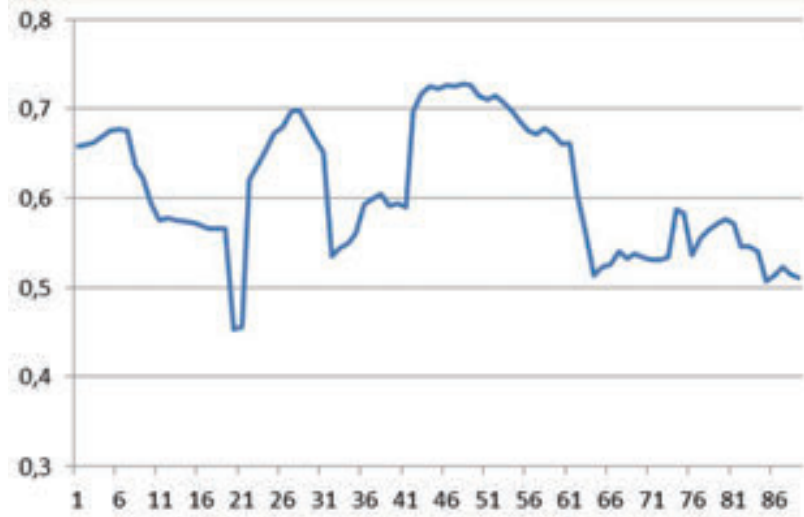

\section{Esempio di profilo superficiale ricavato con profilimetro ad aghi.}

zione del punto di presa. Per far fronte a queste problematiche è stato progettato un treppiede (Figura 30) che consente di migliorare l'operazione.

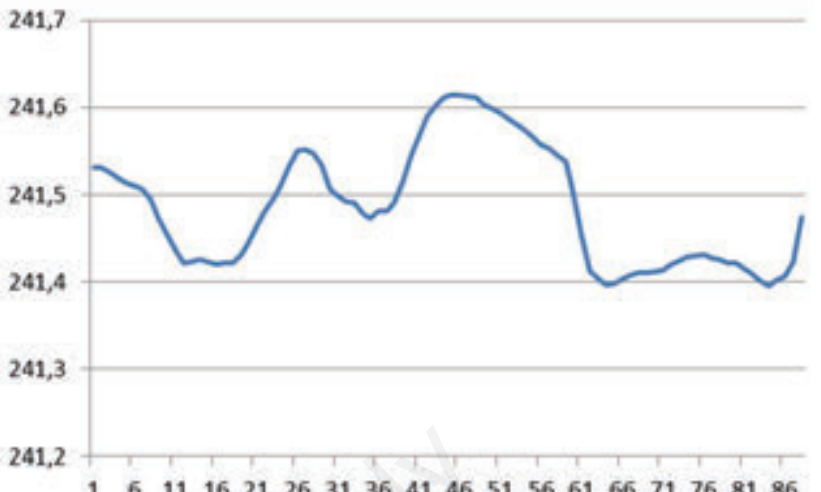

Profilo ricavato dal DEM ERDAS.

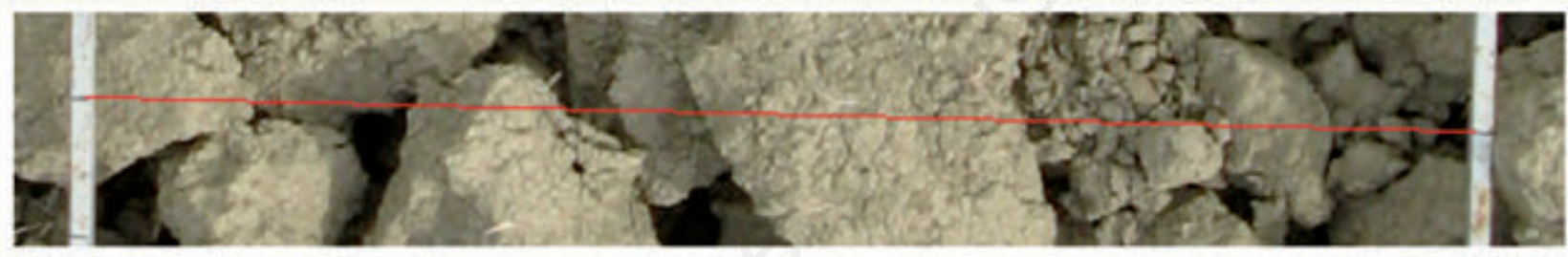

Figura 28. Esempio di una sezione ottenuta con entrambi i metodi.

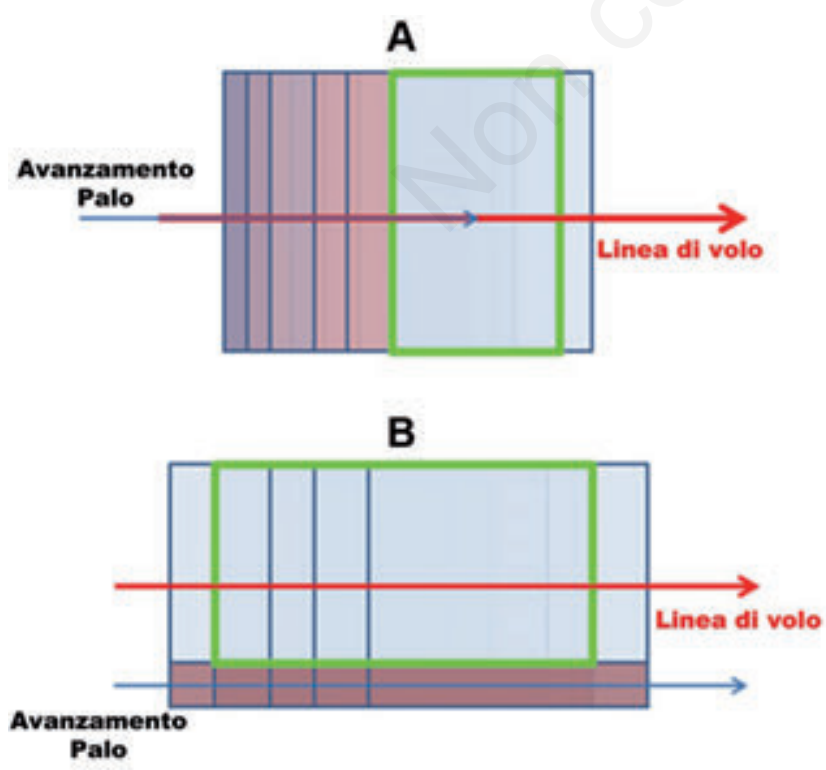

Figura 29. Modalità di avanzamento del palo: A) sulla linea di volo, B)parallelamente alla linea di volo.

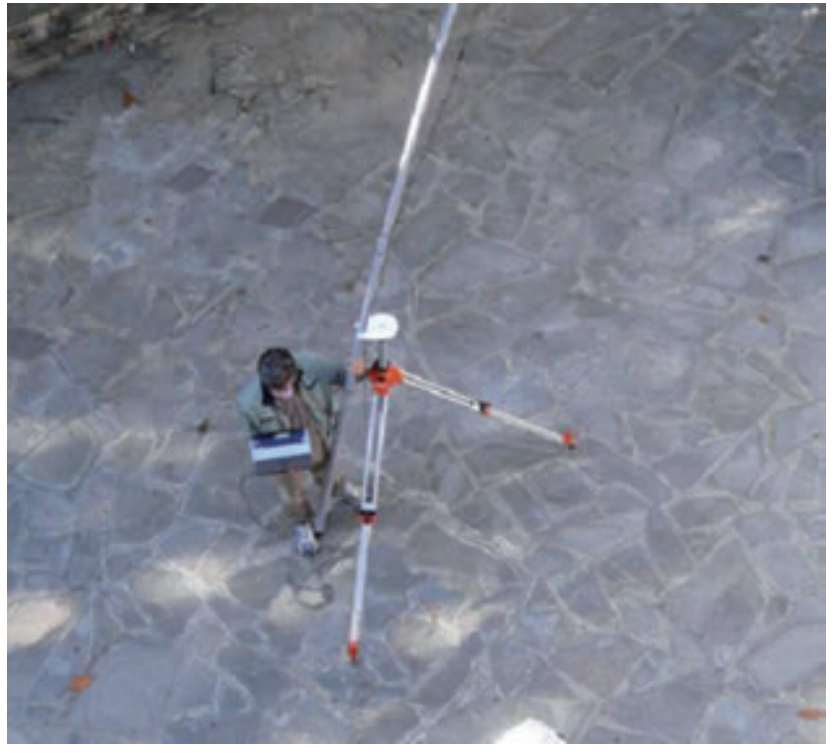

Figura 30. Treppiede realizzato come appoggio per il palo. 
Tabella 4. Indice RR e indice T ricavati con il software MRIC sulle sezioni profilimetriche investigate.

\begin{tabular}{lllll} 
Sezione & RR ERDAS & TR profiligrafo & T profiligrafo \\
6 & 6.71 & 5.30 & 1.56 & 1.83 \\
7 & 9.62 & 8.52 & 1.49 & 1.95 \\
\hline 8 & 6.94 & 6.28 & 1.48 & 2.05 \\
9 & 9.49 & 9.04 & 1.82 & 1.97 \\
10 & 8.65 & 7.84 & 1.69 & 1.88 \\
11 & 8.51 & 7.44 & 1.62 & 1.93 \\
\hline 12 & 12.14 & 11.13 & 1.53 & 1.78 \\
13 & 4.57 & 8.36 & 1.30 & 1.57 \\
14 & 4.57 & 3.49 & 1.30 & 1.49 \\
19 & 9.73 & 9.68 & 1.65 & 1.94 \\
\hline 20 & 5.64 & 8.00 & 1.50 & 1.98 \\
22 & 4.81 & 7.98 & 1.52 & 1.62 \\
\hline 23 & 7.76 & 5.71 & 1.65 & 1.89 \\
24 & 3.86 & 5.19 & 1.27 & 1.78 \\
27 & 5.69 & 6.61 & 1.39 & 1.78 \\
\hline 28 & 5.51 & 6.61 & 1.46 & 2.03 \\
\hline
\end{tabular}

Tabella 5. Test $\boldsymbol{t}$ fra i valori medi di RR rilevati con profiligrafo e con PAP-ERDAS.

\begin{tabular}{lccccccccc} 
& Media & Dv. Std. & N & Diff. & Dv. Std. Diff. & t & gl & \\
RR ERDAS & 6,921 & 2,362 & & & & & \\
RR profiligrafo & 7,301 & 1,801 & 18 & 0,380 & 9 & 0,881 & 17 \\
\hline
\end{tabular}

Tabella 6. Test $t$ fra i valori medi di RR rilevati con profiligrafo e con PAP-ERDAS.

\begin{tabular}{lccccccccc} 
& Media & Dv. Std. & N & Diff. & Dv. Std. Diff. & t & gl & \\
T ERDAS & 1,505 & 0,146 & & & & & \\
T profiligrafo & 1,920 & 0,227 & 18 & 0,414 & 0,289 & $-6,089$ & 17 & 0,000 \\
\hline
\end{tabular}

\section{Conclusioni}

A conclusione delle prove fatte si può dunque dire che ERDAS, con foto scattate con la metodologia PAP in condizioni considerate forse non ottimali, riesce a collimare in maniera corretta i raggi della triangolazione e ad assegnare un valore accettabile alle quote. L'accuratezza delle quote consente di ricavare una rugosità pari a $6,93 \pm 1,17$ che risulta equivalente per i fini pratici di applicazione della modellistica dell'erosione con quella calcolata con il metodo tradizionale del profilo-

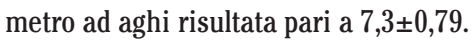

Si può dunque affermare che la metodologia è stata in grado di stimare in maniera deguata la rugosità a costi realmente contenuti soddisfacendo pienamente gli obbiettivi del presente studio.

\section{Bibliografia}

Bazzoffi P, Borselli L, Pellegrini S, Torri D, 2006. Indici di rugosità superficiale del suolo a fini modellistici. In: E.A.C. Costantini (ed.) Metodi di valutazione dei suoli e delle terre. Cantagalli, Siena.
Boiffin J, 1984. La dégradation structurel des couhes superficielles sous l'action des pluies. These de Docteur-ingénieur, Inst. Nat. Agron., Paris, France.

Borselli L, 1998. Soil surface roughness dynamics and its influence on infiltration processes:experimental analysis and modelling. Ph.D. thesis, University of Florence.

Borselli L, 2000. MRIC Multiple Roughness Index Computation: USER Manual. CNR-IGES.

Cogo NP, Moldenhauer WC, Foster GR, 1984. Soil loss reductions from conservation tillage practices. Soil Sci. Soc. Am. J. 48:368-373.

Currence HD, Lovely WG, 1970. The analysis of soil surface roughness. T. ASAE 13:710-714.

Gilley JE, Finkner SC, 1991. Hydraulic roughness coefficient as affected by random roughness. T. ASAE 34:897-903.

Gomarasca MA, 2004. Elementi di geomatica. Associazione Italiana di Telerilevamento, Milano.

Kamphorst EC, Chadoeuf J, Jetten V, Guérif J, 2005. Generating 3D soil surfaces from 2D height measurements to determine depression storage. Catena 62:189-205.

Laflen JM, Foster GR, Onstad CA, 1985. Simulation of individual storm soil loss for modeling the impact of soil erosion on crop productivity. In: S.A. El-Swaify, W.C. Moldenhauer, and A. Lo (eds.), Soil ero- 
sion and conservation. Soil and Water Conservation Society, Ankeny, IO, USA, pp. 285-295.

Mutchler CK, Murphree CE, McGregor K.C. 1982. Subfactor method for computing C-factors for continuous cotton. T. ASAE 25:327-332.

Onstad CA, 1984. Depressional storage on tilled surfaces. T. ASAE 27:729-732.

Renard KG, Foster GR, Weessies GA, Mccool DK, Yoder DC, 1997. Predicting soil erosion by water: a guide to conservation planning with the Revised Universal Soil Loss Equation (RUSLE). Agriculture Handbook 703, US Department of Agriculture.

Salvini R, 2011. Fondamenti di Fotogrammetria. Dispense del corso Master SITT 2011. Università di Siena.

Schwab G0, Frevert RK, Barnes KK, 1981. Soil and water conservation engineering, 3rd ed. J, Wiley \& Sons, New York, NY, USA.

Sumner ME, Stewart BA, 1992. Soil crusting: chemical and physical processes. Lewis Publ., Boca Raton, FL, USA.

Taconet 0, Ciarletti V, 2007. Estimating soil roughness indices on a ridge- and -furrow surface using stereo photogrammetry. Soil Till. Res. 93:64-76.

Verhoeven GJ, 2009. Providing an archaeological bird's-eye view- an overall picture of ground - based means to execute Low-altitude Aerial Photography (LAAP) in archaeology. Archaeol. Prospect. 16:233-249.

Weltz MA, Renard KG, Simanton JR, 1987. Revised universal soil loss equation for western rangelands. pp 104-111 in US/Mexico Symp. Strategies for classification and management of native vegetation for food production in arid zones. Tucson, AZ, USA.

Wischmeier WH, 1975. Estimating the soil boss equations cover and management factor for undisturbed lands. pp 118-125 in Proc. of the Sediment-Yield Workshop, Washington, DC, USA.

Wischmeier WH, Smith DD, 1978. Predicting rainfall erosion losses, a guide to conservation planning. Agriculture Handbook 537. US Department of Agriculture, Washington, DC, USA. 